Review

\title{
Fair Look at Coordination Oligomerization of Higher $\alpha$-Olefins
}

\author{
Ilya Nifant'ev *(i) and Pavel Ivchenko *(D) \\ A.V. Topchiev Institute of Petrochemical Synthesis RAS, 29 Leninsky Pr., 119991 Moscow, Russia \\ * Correspondence: ilnif@yahoo.com (I.N.); phpasha1@yandex.ru (P.I.)
}

Received: 20 April 2020; Accepted: 30 April 2020; Published: 9 May 2020

\begin{abstract}
Coordination catalysis is a highly efficient alternative to more traditional acid catalysis in the oligomerization of $\alpha$-olefins. The distinct advantage of transition metal-based catalysts is the structural homogeneity of the oligomers. Given the great diversity of the catalysts and option of varying the reaction conditions, a wide spectrum of processes can be implemented. In recent years, both methylenealkanes (vinylidene dimers of $\alpha$-olefins) and structurally uniform oligomers with the desired degrees of polymerization have become available for later use in the synthesis of amphiphilic organic compounds and polymers, high-quality oils or lubricants, and other prospective materials. In the present review, we discussed the selective dimerization and oligomerization of $\alpha$-olefins, catalyzed by metallocene and post-metallocene complexes, and explored the prospects for the further applications of the coordination $\alpha$-olefin dimers and oligomers.
\end{abstract}

Keywords: dimerization; metallocenes; oils; oligomerization; single-site catalysts; polymerization

\section{Introduction}

The achievement of the best characteristics of chemical product by the use of efficient knowledge-intensive technologies is the most productive avenue for achieving real development goals in the modern chemical industry. The Ziegler-Natta polymerization of $\alpha$-olefins [1-5] is an excellent example of such technologies that provides humanity with $\sim 2 \times 10^{8}$ tons of plastics per year. Ethylene and propylene are raw materials for the modern polyolefin industry; thousands of articles and hundreds of reviews are devoted to the coordination homopolymerization and copolymerization of these monomers. The polymerization and oligomerization of higher $\alpha$-olefins have been studied less intensively. The oligomerization of $C_{8}+\alpha$-olefins followed by hydrogenation to form engine Group 4 poly- $\alpha$-olefin oil (PAO) base stocks and lubricants (Scheme 1a) [6,7], and the synthesis of ultra-high MW polyolefins (Scheme 1b) as drag reducing agents [8,9], were actual topics of the applied research.

It was the Group 4 oil industry that led to the growing interest in the oligomerization of higher $\alpha$-olefins in the mid-20th century $[10,11]$. The conventional technologies of acid-catalyzed oligomerization with the use of $\mathrm{BF}_{3} / \mathrm{ROH}$ or $\mathrm{Al}$ chloride catalysts remains relevant for the production of the lower oligomers of $\alpha$-olefins [12,13]. The cationic oligomerization is accompanied by huge numbers of rearrangements $[14,15]$. However, lower $\alpha$-olefin oligomers obtained by cationic oligomerization were exceedingly defined as structurally uniform reaction products $[16,17]$. Moreover, most of the recent publications devoted to the oligomerization of higher $\alpha$-olefins have discussed acid-catalyzed processes [18-32].

The review of Nicholas [7] was also focused on cationic polymerization; the mini-review of Ray et al., devoted to the synthesis of PAOs [12], addresses only part of the problem of coordination polymerization. The reviews of Janiak [33,34] and Belov [35] were focused on the coordination oligomerization of $\alpha$-olefins, but these works, published more than 10 years ago, are now objectively outdated. 
(a)<smiles>[R]CC=C</smiles>

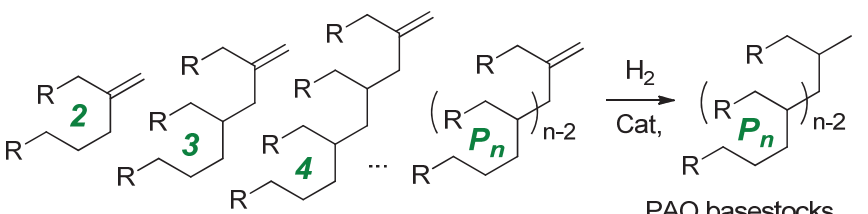

(b)

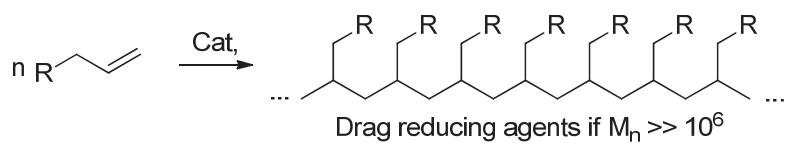

Scheme 1. Coordination oligomerization (a) and polymerization (b) of $\alpha$-olefins.

Taking into account the fact that the selective coordination oligomerization of $\alpha$-olefins is a growth point of the actual petrochemical industry [7], in the present review, we tried to organize the current scientific information on that topic. In our review, we discussed the plausible mechanisms of the coordination dimerization and oligomerization of $\alpha$-olefins, collated the data on the catalytic properties of different single-site catalysts, and discussed the problem of the catalyst design. Additionally, we were to focus on looking at the relationship between molecular structure and the characteristics of the oligomers of $\alpha$-olefins, and on the prospects of the application of these dimers and oligomers in the synthesis of amphiphilic organic compounds and polymers, in the production of fuels, oils, lubricants, and other actual products.

\section{Coordination Dimerization of $\alpha$-Olefins}

\subsection{Group 4 Metallocene-Catalyzed Synthesis of Methylenealkanes}

The zirconocene-catalyzed dimerization of $\alpha$-olefins (Scheme 2) has been known since the late 1980s [36]. The reaction proceeds in the presence of zirconocene dichloride $\left(\eta^{5}-\mathrm{C}_{5} \mathrm{H}_{5}\right)_{2} \mathrm{ZrCl}_{2}(\mathbf{1}$, Scheme 2a), activated by minimal amounts of methylalumoxane (MAO) and results in the selective formation of methylenealkanes, olefins containing the vinylidene fragment $>\mathrm{C}=\mathrm{CH}_{2}$. The prospects of the synthetic use of this reaction in the synthesis of methylenealkanes were studied by Christoffers and Bergman for linear $\alpha$-olefins, allylbenzene, and 1,2-diallylbenzene [37,38], and by Erker et al. for 1,5-hexadiene and 1,6-heptadiene [39] (Scheme 2a). The reaction was complicated by the isomerization of the starting $\alpha$-olefins, by the formation of higher oligomers, and by the deactivation of the catalyst.

Nifant'ev et al. optimized this approach significantly. Based on the results of $X$-ray diffraction studies of bis(cyclopentadienyl) zirconium complexes 1-6 (Scheme 3), they proposed the structure of highly active and selective pre-catalyst 6 . The use of 6 activated by $\sim 20$ equivalents (eq.) of triisobutylalumnium (TIBA) and 10 eq. of methylalumoxane (MAO) provided 92\%-94\% yields of vinylidene dimers of linear $\alpha$-olefins [40-43]. As compared with zirconocene 1, in the presence of the 6 dimer of sterically hindered olefin, 3-methylbut-1-ene was obtained with an average yield; substituted silanes and allylthiophenes also formed vinylidene dimers [41] (Scheme 2b). Note that the zirconium pre-catalysts 7 [44-46], 8 [47], 3 [48], 9 [49,50], and 10 [51] and the hafnium complex 11 [48], studied previously in $\alpha$-olefin dimerization, were less active and selective in comparison with $\mathbf{6}$.

The replacement of the activator of the zirconocene pre-catalyst by perfluoroaryl borate resulted in the migration of the $\mathrm{C}=\mathrm{C}$ bond: thus, in the presence of dimethyl zirconocene 12 (Scheme 3), activated by $[\mathrm{PhNMe} 2 \mathrm{H}]\left[\mathrm{B}\left(\mathrm{C}_{6} \mathrm{~F}_{5}\right)_{4}\right]$, 1-decene formed trisubstituted $\mathrm{C}_{20}$ olefins, and the yield of 9-methylenenonadecane was only 30\% [52,53]. Tens of Group 4 metallocenes were studied in the coordination oligomerization of $\alpha$-olefins (Scheme 3, Section 3); however, dimer fractions were the main reaction products only for the first dozen, 1-12.

The mechanism of the zirconocene-catalyzed dimerization of $\alpha$-olefins remains unclear in terms of detail. On the surface, this mechanism is not very different from the conventional cationic mechanism, and the high selectivity of dimerization can be explained by the low value of the activation barrier of $\beta$-hydride transfer (or $\beta$-hydride elimination) after the insertion of the second molecule of monomer 
in comparison with the activation energy of the chain propagation. However, this interpretation does not match the results of the catalytic experiments. As far back as the 1990s [37,38], Christoffers and Bergman demonstrated that chloride is an indispensable component of the catalyst system that provides the high selectivity of dimerization; the special role of $\mathrm{Zr}-\mathrm{Cl}$ bonding was also proposed by Hessen et al. [54]. Such an effect was confirmed experimentally by an increase in the selectivity of zirconocene/MAO-catalyzed dimerization in the presence of $\mathrm{R}_{2} \mathrm{AlCl}[40,55,56]$, and by the formation of oligomers when $\mathrm{Cp}_{2} \mathrm{ZrMe}_{2} / \mathrm{B}\left(\mathrm{C}_{6} \mathrm{~F}_{5}\right)_{3}$ was used [57]. To explain the experimental facts, Nifant'ev et al. proposed a binuclear $\mathrm{Zr}-\mathrm{Al}$ mechanistic concept $[40,41]$ involving the $\mathrm{Zr}-(\mu-\mathrm{Cl})(\mu-\mathrm{H}) \mathrm{AlR}_{2}$ catalytic species (Scheme $2 \mathrm{c}$ ). The early studies of the interaction of $\mathbf{1}$ with TIBA and $\mathrm{HAl}\left({ }^{\mathrm{i}} \mathrm{Bu}\right)_{2}$ [58-61] were the experimental basis of this concept.

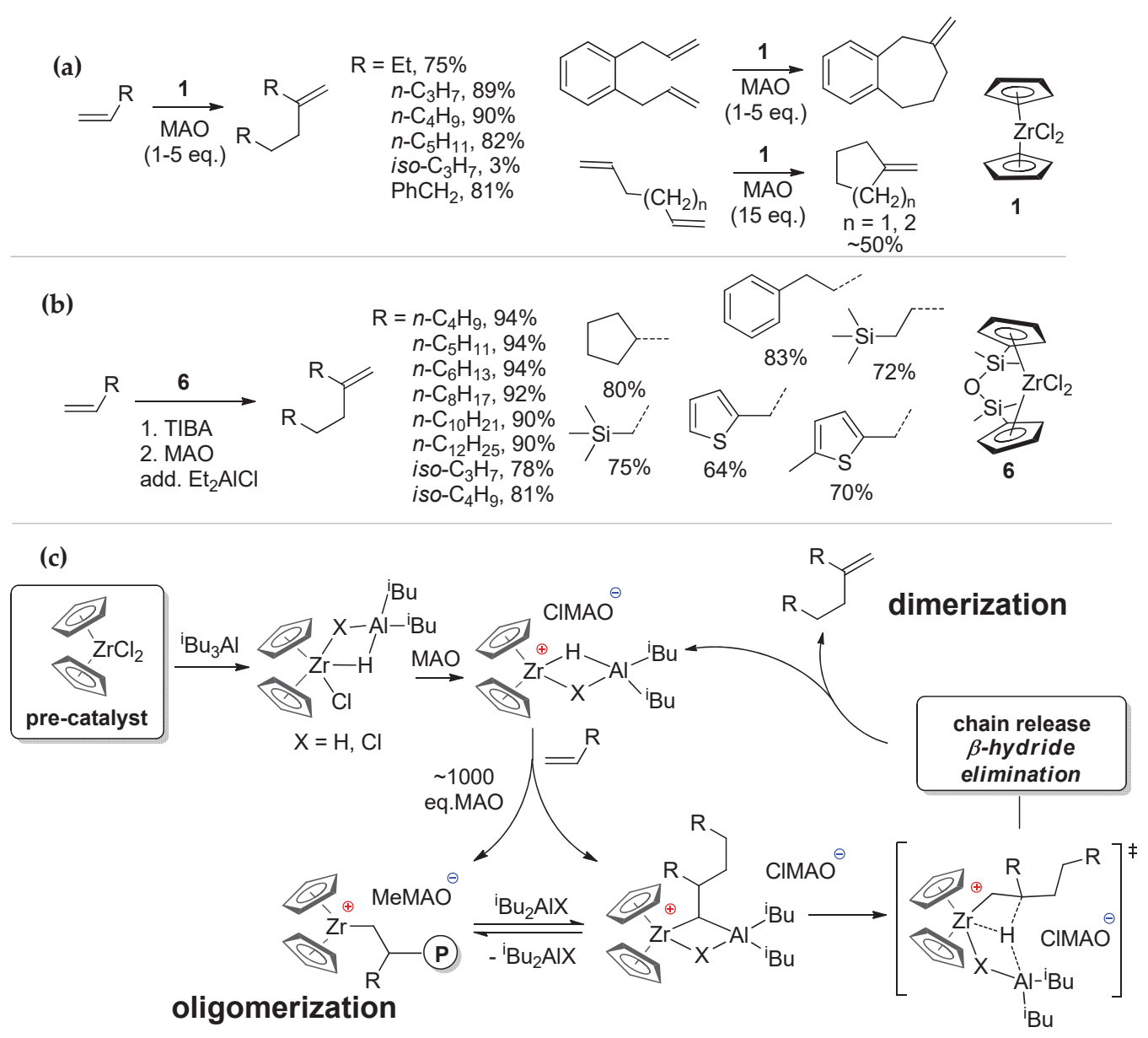

Scheme 2. Early works on the zirconocene-catalyzed dimerization of $\alpha$-olefins [37-39] (a); high efficiency of the pre-catalyst $\mathbf{6}[40,41]$ (b); cationic $\mathrm{Zr}-\mathrm{Al}$ catalytic species proposed to explain the reactivity of zirconocene under low $\mathrm{MAO} / \mathrm{Zr}$ ratios $[40,41]$ (c).

Recently, this $\mathrm{Zr}-\mathrm{Al}$ binuclear mechanism was studied theoretically for propylene oligomerization [62]. Using the quantum chemical modeling at the M-06x/DGDZVP level of the density functional theory (DFT), the qualitative difference between mononuclear and binuclear mechanisms (for $\left[\left(\eta^{5}-\mathrm{C}_{5} \mathrm{H}_{5}\right)_{2} \mathrm{Zr}-\mathrm{Alkyl}\right]^{+}$and $\left[\left(\eta^{5}-\mathrm{C}_{5} \mathrm{H}_{5}\right)_{2} \mathrm{Zr}-\mathrm{Alkyl}\left(\mathrm{R}_{2} \mathrm{AlX}\right)\right]^{+}$species, respectively), was demonstrated. Without $\mathrm{R}_{2} \mathrm{AlX}$ coordination (mononuclear mechanism), oligomerization was found to be a preferable reaction pathway. In binuclear complexes $(X=\mathrm{H}, \mathrm{Cl})$, the formation of vinylidene dimers had been greatly facilitated by an explicit $\mathrm{Zr}-\mathrm{Al}$ cooperative effect (Figure 1). 

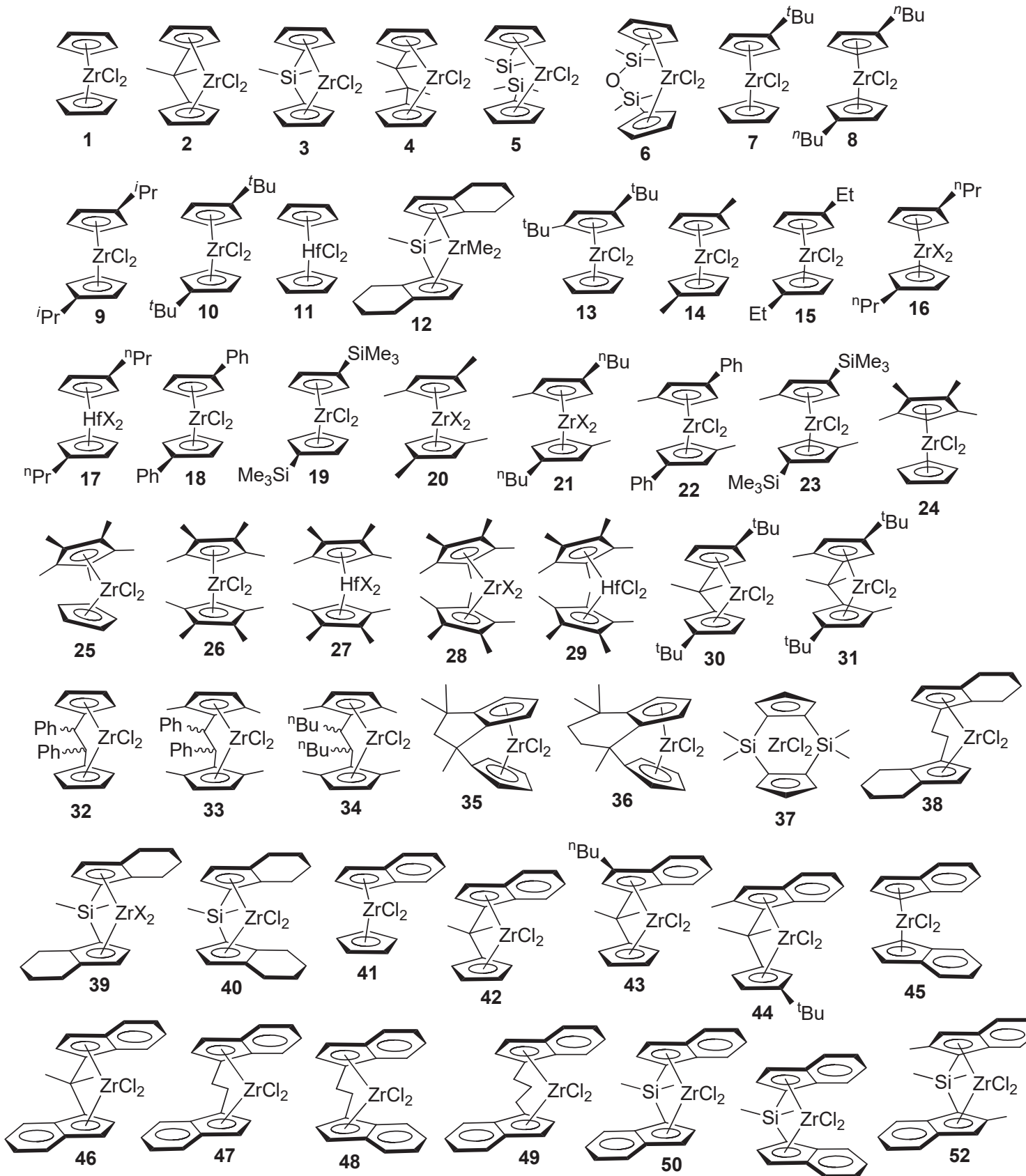
$44^{\mathrm{t} B u}$

45 24
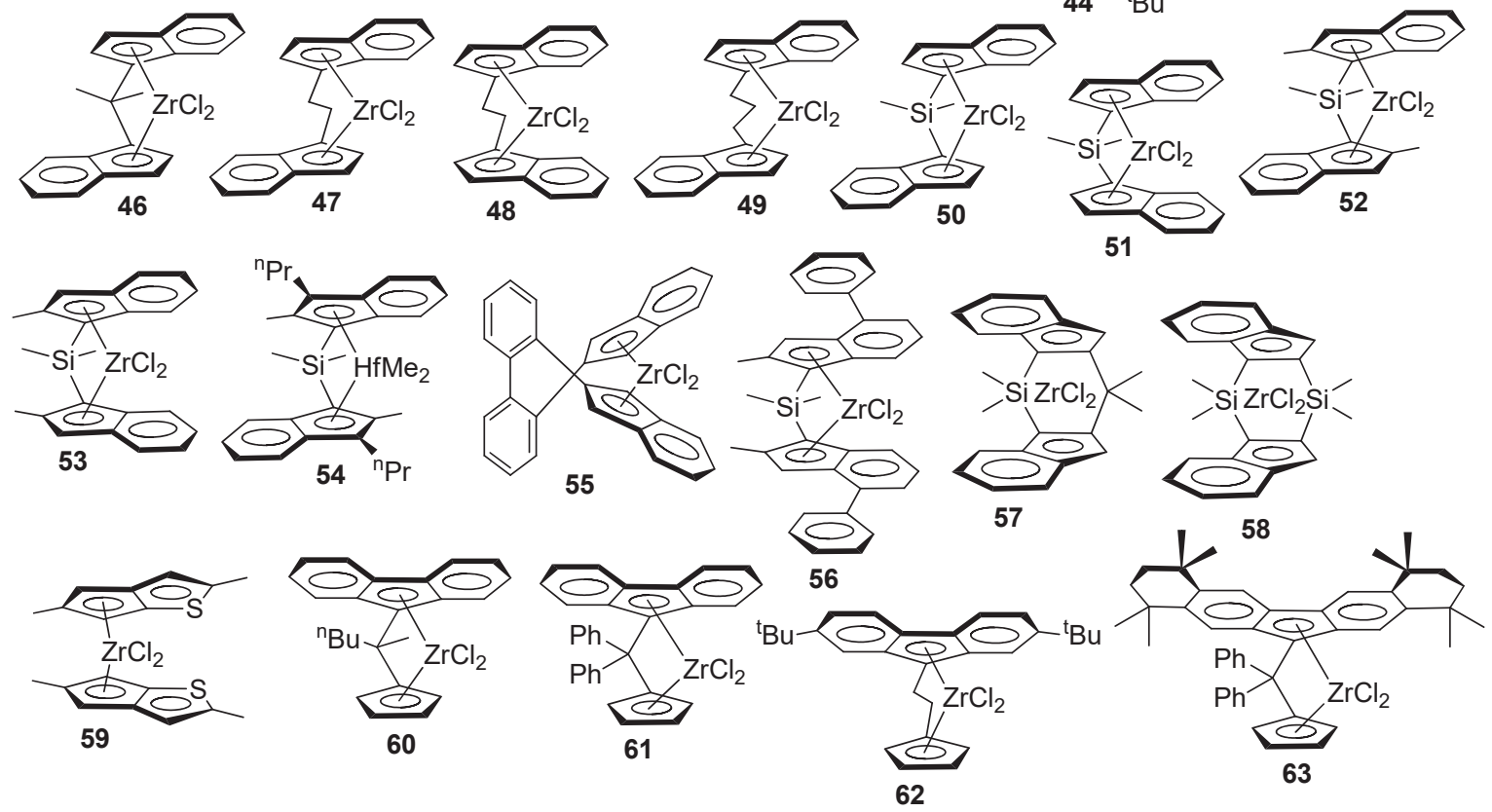

Scheme 3. Metallocene pre-catalysts studied in the coordination oligomerization of $\alpha$-olefins. 

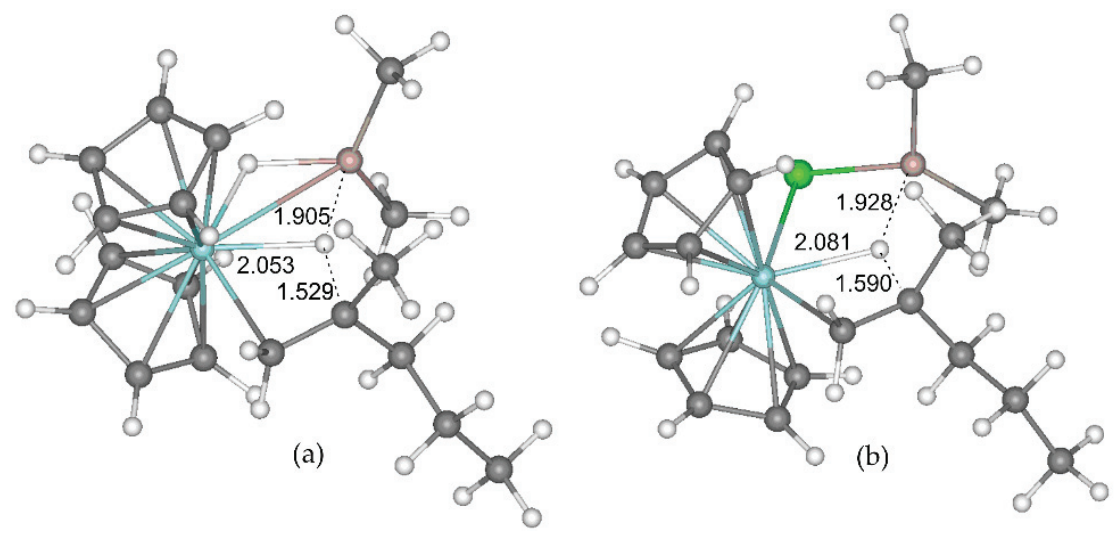

Figure 1. Cooperative effect in $\mathrm{Zr}-\mathrm{Al}$ transition states of the $\beta$-hydride elimination stage in the framework of the $\mathrm{Zr}-\mathrm{Al}$ binuclear mechanism [62]. Copyright (2019) MDPI.

\subsection{Dimerization of $\alpha$-Olefins Catalyzed by Other Complexes of Transition and Rare-Earth Metals}

A number of complexes-namely, $\mathrm{WCl}_{6} / \mathrm{Et}_{2} \mathrm{AlCl}$ [63], zirconium and hafnium adducts with tetradentate ligand 64 [64], half-sandwich complexes of Ta 65 [65,66] and Co 66 [67], a bis-indenyl complex of Y 67 [68], a zwitter-ionic zirconium complex 69 [54], and a sandwich hydride complex of Sc 68 [69] -were studied in the coordination dimerization of $\alpha$-olefins (Scheme 4a). In the presence of a $\mathrm{WCl}_{6}$-based catalyst, a mixture of vinylidene olefins was formed (Scheme $4 \mathrm{~b}$ ); similar mixtures were obtained in the presence of $\mathbf{6 5}$. The half-sandwich complex $\mathbf{6 6}$ catalyzed the formation of dimers containing significant amounts of linear olefins. The sandwich complexes of rare-earth metals 67 and 68 , as well as the zwitter-ionic complex 69 demonstrated moderate catalytic activity and high selectivity in the formation of the single reaction product; however, the catalytic performance of these complexes dramatically decreased over time (TON 100). Among the complexes mentioned above, only $64 \mathrm{Hf}(\mathrm{R}=\mathrm{nBu})$ was capable of beating zirconocenes on the criteria of selectivity and catalytic productivity; however, its stability was much lower.

(a)
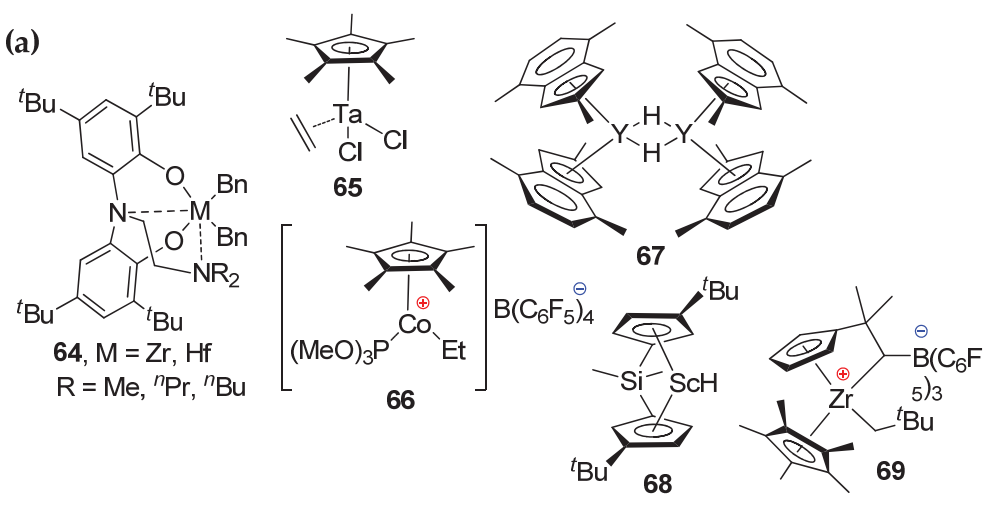

(b)

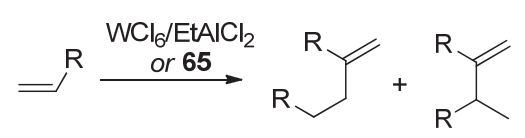

Scheme 4. Other metal complexes that are active in the selective oligomerization of $\alpha$-olefins (a); formation of vinylidene olefins in the presence of $\mathrm{W}$ and Ta complexes (b).

\section{Coordination Oligomerization of $\alpha$-Olefins}

\subsection{Common Aspects of the Coordination Oligomerization of $\alpha$-Olefins}

Different types of transition metal complex were studied in the coordination oligomerization of $\alpha$-olefins. Traditional Ziegler-Natta catalysts demonstrated low activity $[70,71]$ and will not be 
discussed in our review. Group 4 metal sandwich complexes, metallocenes, are the most efficient and most extensively researched catalysts for this process. Scheme 5 illustrates the main and side reactions observed during metallocene-catalyzed oligomerization. The degree of polymerization, $D P_{n}$, is related to a variety of factors, such as the molecular structure of metallocene, type and quantity of the activator, reaction temperature, and presence of the molecular hydrogen. In the heyday of metallocene catalysis (1990s-early 2000s), the zirconocene-catalyzed polymerization of higher $\alpha$-olefins was largely viewed as a model process for the study of the mechanism of chain propagation and chain release [72-79].

A: Zr-Al transfer, $-\mathrm{CH}_{2} \mathrm{OH}$ detected B: $\beta$-hydride elimination, $>\mathrm{C}=\mathrm{CH}_{2}$ detected C: $\beta$-alkyl elimination, $-\mathrm{C} \underline{\mathrm{H}}=\mathrm{CH}_{2}$ detected D: $1,2-\mathrm{Zr}$ shift or allyl formation, $>\mathrm{C}=\mathrm{C}$ ㅂ- - detected

E: 2,1-insertion and $\beta$-hydride elimination, $-\mathrm{C} \underline{\mathrm{H}}=\mathrm{C} \underline{\mathrm{H}}$ - detected
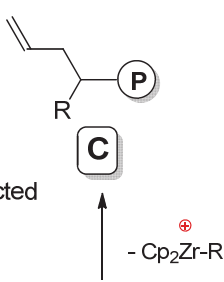

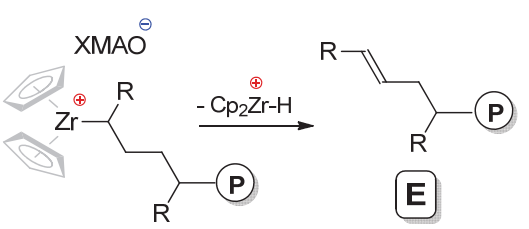

2,1-insertion product

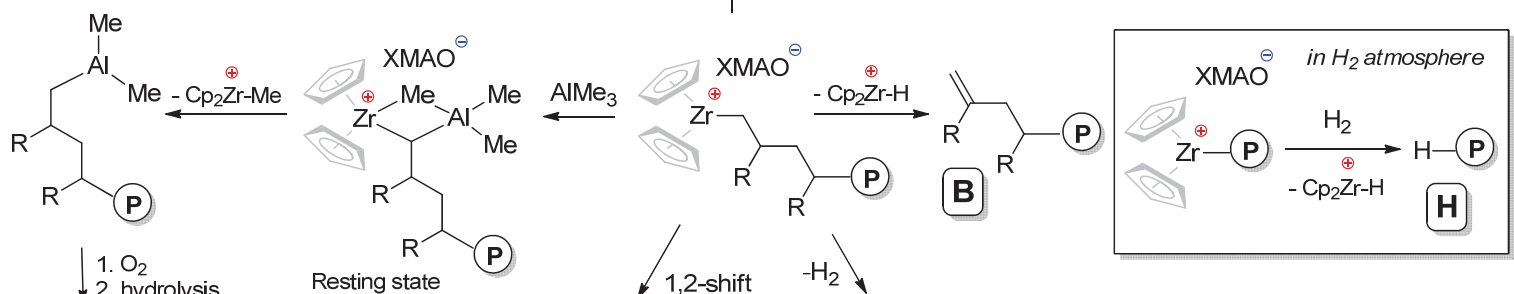
2. hydrolysis Resting state<smiles>[R]C(C)CC([R])CO</smiles>

A

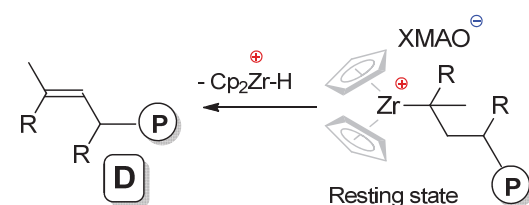

Resting state

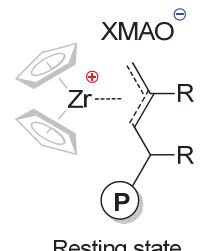

Resting state

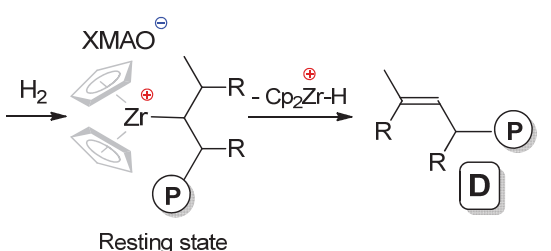

Scheme 5. Traditional mechanisms of chain release in $\alpha$-olefin polymerization with a formation of characteristic chain-end groups (routes A-E) and saturated polyolefin species (route H) $[72,73,80]$.

Usually, the zirconocene-catalyzed oligomerization and polymerization of higher $\alpha$-olefins was studied in the laboratory using the activation of $\mathrm{LZrCl}_{2}$ pre-catalysts by MAO with extremely high $\mathrm{Al} / \mathrm{Zr}$ ratios $\left(10^{3}-10^{4}\right)$, or the activation of $\mathrm{LZrMe}_{2}$ pre-catalysts by perfluoroaryl borates. Many of the zirconocenes under these conditions catalyzed the formation of high MW polyolefins $\left(M_{\mathrm{n}} \sim 10^{4}-10^{5} \mathrm{Da}\right)$; however, several complexes were efficient in the synthesis of oligomers (see below, Section 3.2). High $\mathrm{Al}_{\mathrm{MAO}} / \mathrm{Zr}$ ratios facilitate the formation of $\mathrm{Zr}-\mathrm{Al}$ alkyl complexes [81-85] that are unable to perform monomer coordination. Such complexes are intermediates of the chain release via $\mathrm{Zr}-\mathrm{Al}$ transfer [86-88]. At the same time, the practice of using high $\mathrm{Al}_{\mathrm{MAO}} / \mathrm{Zr}$ ratios in the laboratory was due to lthe ow effectiveness of MAO as an alkylation agent; the $\mathrm{Al}_{\mathrm{MAO}} / \mathrm{Zr}$ ratios of $10^{2} \mathrm{MAO}$ were found to be insufficient for the activation of bis-indenyl and bis-fluorenyl complexes [89]. In 2017, Nifant'ev et al. proposed a two-stage activation method for a wide array of $\mathrm{LZrCl}_{2}$ complexes. This method was based on the reaction of $\mathrm{LZrCl}_{2}$ with TIBA (formation of $\mathrm{Zr}-\mathrm{Al}$ alkyl-hydrides) followed by the reaction with $~ 10$ eq. of aMAO; dozens of zirconocene dichlorides were studied in such industrially important conditions [40,80,90-92] (see Section 3.3). In contrast to dimerization, the activation of zirconocenes by perfluoroaryl borates was successfully used in the oligomerization of higher $\alpha$-olefins (see Section 3.4). The formulas of the metallocenes studied in the oligomerization of $\alpha$-olefins are presented in Scheme 3.

\subsection{Metallocene-Catalyzed Oligomerization of $\alpha$-Olefins and Activation by $10^{2}-10^{3}$ eq. $M A O$}

Dozens of scientific articles and patents have been devoted to the oligomerization of higher $\alpha$-olefins, catalyzed by $\mathrm{Zr}$ and $\mathrm{Hf}$ sandwich complexes and activated by a large excess of MAO (Table 1 ). The main target of the studies, namely, the synthesis of Group 4 oil base stocks, required the use of 
specific types of product characteristic such as the kinematic viscosity at a given temperature, $\mathrm{KV}^{\mathrm{t}}\left(\mathrm{KV}^{100}\right.$ are given in Table 1), and viscosity index, VI, as an alternative to the degree of polymerization, $D P_{n}$, or oligomer distribution.

Table 1. Metallocene-catalyzed oligomerization of $\alpha$-olefins at high $\mathrm{Al} / \mathrm{Zr}$ ratios

\begin{tabular}{|c|c|c|c|c|c|c|c|c|c|c|c|}
\hline \multirow[t]{2}{*}{ Cat. } & \multirow[t]{2}{*}{ Mon. } & \multirow[t]{2}{*}{ Mon]/[Zr] } & \multirow{2}{*}{$\begin{array}{l}{[\mathrm{Al}] /} \\
{[\mathrm{Zr}]}\end{array}$} & \multirow{2}{*}{$\begin{array}{l}\mathrm{T}, \\
{ }^{\circ} \mathrm{C}\end{array}$} & \multirow{2}{*}{$\begin{array}{l}\mathrm{H}_{2} \\
\text { bar }\end{array}$} & \multirow[t]{2}{*}{ TOF, $h^{-1}$} & $D P_{\mathrm{n}}$ & $K V^{1001}$ & $V I^{2}$ & $\begin{array}{c}\text { Additional } \\
\text { Remarks }\end{array}$ & \multirow[t]{2}{*}{ Ref. } \\
\hline & & & & & & & \multicolumn{4}{|c|}{ or Oligomer Distribution for $D P_{n} 2,3,4,5$} & \\
\hline \multirow{10}{*}{1} & $\mathrm{C}_{6}$ & $1.3 \times 10^{5}$ & 4000 & 50 & - & $5.9 \times 10^{4}$ & 4.9 & - & - & - & {$[45,46]$} \\
\hline & $\mathrm{C}_{6}$ & $2 \times 10^{3}$ & 200 & 60 & - & - & \multicolumn{4}{|c|}{$\%$ olig. $45(2): 20(3-5)$, res. $21^{3}$} & [91] \\
\hline & $\mathrm{C}_{8}$ & $2 \times 10^{3}$ & 200 & 60 & - & - & \multicolumn{4}{|c|}{ \% olig. $45(2): 25(3-5)$, res. 15} & {$[91]$} \\
\hline & $\mathrm{C}_{10}$ & $1.0 \times 10^{5}$ & 1000 & 40 & - & $1.1 \times 10^{4}$ & - & 41 & 196 & - & [93] \\
\hline & $C_{10}$ & $1.0 \times 10^{5}$ & 1000 & 80 & - & $3.2 \times 10^{4}$ & - & 2.5 & 181 & - & [93] \\
\hline & $\mathrm{C}_{10}$ & $3.0 \times 10^{4}$ & 30 & 50 & 1 & $2.9 \times 10^{4}$ & \multicolumn{4}{|c|}{$\%$ olig. 42:11:17:5, res. 35} & [51] \\
\hline & $\mathrm{C}_{10}$ & $5.0 \times 10^{4}$ & 300 & 70 & - & $9.2 \times 10^{3}$ & - & 17 & 167 & $24 \%$ of dimer & [49] \\
\hline & $\mathrm{C}_{10}$ & $5.0 \times 10^{4}$ & 300 & 110 & - & $3.7 \times 10^{3}$ & - & 5.9 & 152 & $55 \%$ of dimer & [49] \\
\hline & $\mathrm{C}_{14}$ & $1.6 \times 10^{3}$ & 530 & 40 & - & $1.1 \times 10^{3}$ & \multicolumn{4}{|c|}{$\%$ olig. $42: 25: 16: 8$, res. 6} & {$[50]$} \\
\hline & $\mathrm{C}_{14}$ & $1.6 \times 10^{3}$ & 530 & 60 & - & $>2 \times 10^{3}$ & \multicolumn{4}{|c|}{ \% olig. $60: 23: 8: 4$, res. 5} & [50] \\
\hline 3 & $\mathrm{C}_{10}$ & $1.0 \times 10^{5}$ & 1000 & 40 & - & $1.1 \times 10^{4}$ & - & $2460^{\circ}$ & 344 & \multirow{3}{*}{-} & [93] \\
\hline \multirow[t]{5}{*}{7} & $\mathrm{C}_{6}$ & $1.3 \times 10^{5}$ & 4000 & 50 & - & $9.4 \times 10^{4}$ & 5.7 & - & - & & {$[45,46]$} \\
\hline & $\mathrm{C}_{6}$ & $1.3 \times 10^{5}$ & 4000 & 50 & - & $2.0 \times 10^{4}$ & 3.9 & - & - & & {$[45,46]$} \\
\hline & $\mathrm{C}_{6}$ & $2 \times 10^{3}$ & 200 & 60 & - & - & & $\%$ olig. 15 & 2):30 (3 & ), res. 41 & [91] \\
\hline & $\mathrm{C}_{8}$ & 500 & 200 & 60 & - & - & & $\%$ olig. 31 & 2):41 (3 & ), res. 16 & [91] \\
\hline & $\mathrm{C}_{10}$ & $1.0 \times 10^{5}$ & 1000 & 90 & - & $6.3 \times 10^{4}$ & - & 2.3 & 163 & & [93] \\
\hline 8 & $\mathrm{C}_{10}$ & $3.0 \times 10^{4}$ & 30 & 50 & 1 & $2.7 \times 10^{4}$ & & $\%$ olig. 4 & $26: 11$ & res. 12 & [51] \\
\hline 8 & $\mathrm{C}_{10}$ & $3.0 \times 10^{4}$ & 100 & 50 & 1 & $>3 \times 10^{4}$ & & $\%$ olig. 4 & $: 27: 11$ & res. 13 & [51] \\
\hline & $\mathrm{C}_{10}$ & $3.0 \times 10^{4}$ & 300 & 50 & 1 & $2.8 \times 10^{4}$ & & $\%$ olig. & $4: 28: 9$ & res. 6 & [51] \\
\hline & $\mathrm{C}_{10}$ & $3.0 \times 10^{4}$ & 600 & 50 & 1 & $2.9 \times 10^{4}$ & & $\%$ olig. 2 & $: 28: 16$ & res. 20 & [51] \\
\hline & $C_{10}$ & $5.0 \times 10^{4}$ & 300 & 110 & - & $6.0 \times 10^{3}$ & - & $6.7^{\circ}$ & 156 & $49 \%$ of dimer & [49] \\
\hline & $\mathrm{C}_{10}$ & $5.0 \times 10^{4}$ & 300 & 110 & - & $6.0 \times 10^{3}$ & - & 6.7 & 156 & $49 \%$ of dimer & [49] \\
\hline & $\mathrm{C}_{10}$ & $2.7 \times 10^{3}$ & 340 & 50 & - & $>3 \times 10^{3}$ & & $\%$ olig. 4 & $24: 11$ & res. 17 & [50] \\
\hline 9 & $\mathrm{C}_{10}$ & $3.0 \times 10^{4}$ & 30 & 50 & 1 & $1.6 \times 10^{4}$ & & $\%$ olig. & 8:12:8: & res. 46 & [51] \\
\hline & $\mathrm{C}_{10}$ & $5.0 \times 10^{4}$ & 300 & 110 & - & $6.0 \times 10^{3}$ & - & $5.7^{\circ}$ & 152 & $50 \%$ of dimer & [49] \\
\hline 13 & $\mathrm{C}_{6}$ & $1.3 \times 10^{5}$ & 4000 & 50 & - & $3.2 \times 10^{4}$ & 14 & - & - & & {$[45,46]$} \\
\hline 14 & $\mathrm{C}_{10}$ & $5.0 \times 10^{4}$ & 300 & 110 & - & $6.1 \times 10^{3}$ & - & 8.2 & 159 & $40 \%$ of dimer & [49] \\
\hline 19 & $C_{10}$ & $3 \times 10^{4}$ & 30 & 50 & 1 & $1.5 \times 10^{4}$ & & $\%$ olig. 2 & $24: 10$ & res. 25 & [51] \\
\hline & $\mathrm{C}_{10}$ & $5.0 \times 10^{4}$ & 300 & 70 & - & $1.1 \times 10^{4}$ & - & 61 & 190 & $6 \%$ of dimer & [49] \\
\hline 20 & $\mathrm{C}_{10}$ & $5.0 \times 10^{4}$ & 300 & 110 & - & $9.8 \times 10^{3}$ & - & 17 & 162 & $15 \%$ of dimer & [49] \\
\hline 23 & $\mathrm{C}_{10}$ & $3.0 \times 10^{4}$ & 30 & 50 & 1 & $8 \times 10^{3}$ & & $\%$ olig. & $3: 5: 4: 3$ & es. 75 & [51] \\
\hline 24 & $\mathrm{C}_{6}$ & $1.3 \times 10^{5}$ & 4000 & 50 & - & $7.5 \times 10^{4}$ & 14 & - & - & & {$[45,46]$} \\
\hline 25 & $\mathrm{C}_{6}$ & $1.3 \times 10^{5}$ & 4000 & 50 & - & $3.9 \times 10^{4}$ & 45 & - & - & & {$[45,46]$} \\
\hline & $\mathrm{C}_{6}$ & $1.3 \times 10^{5}$ & 4000 & 50 & - & $6.5 \times 10^{4}$ & 83 & - & - & & {$[45,46]$} \\
\hline 26 & $\mathrm{C}_{10}$ & $5.0 \times 10^{4}$ & 300 & 70 & - & $1.2 \times 10^{4}$ & - & 154 & - & $1 \%$ of dimer & [49] \\
\hline $28, X$ & $\mathrm{C}_{6}$ & $1.3 \times 10^{5}$ & 4000 & 50 & - & $3.4 \times 10^{4}$ & 19 & - & - & & {$[45,46]$} \\
\hline$=\mathrm{Cl}$ & $C_{10}$ & $5.0 \times 10^{4}$ & 300 & 70 & - & $2.3 \times 10^{3}$ & - & 115 & 224 & $5 \%$ of dimer & [49] \\
\hline 37 & $\mathrm{C}_{6}$ & $1.0 \times 10^{3}$ & 500 & 25 & - & 630 & $\sim 3$ & & $49 \%$ & dimer & [94] \\
\hline & $\mathrm{C}_{10}$ & $1.5 \times 10^{5}$ & 1000 & 80 & - & $8.3 \times 10^{4}$ & 26 & 101 & - & & [95] \\
\hline 38 & $\mathrm{C}_{10}$ & $1.5 \times 10^{5}$ & 1000 & 80 & 5 & $8.2 \times 10^{4}$ & 14 & 28 & - & & [95] \\
\hline & $\mathrm{C}_{10}$ & $1.5 \times 10^{5}$ & 1000 & 80 & 15 & $1.3 \times 10^{5}$ & 12 & 32 & - & & [95] \\
\hline 41 & $\mathrm{C}_{10}$ & $1.0 \times 10^{5}$ & 250 & 100 & - & $1.1 \times 10^{4}$ & 72 & - & - & & [96] \\
\hline 42 & $\mathrm{C}_{8}$ & $1.7 \times 10^{5}$ & 1000 & 25 & 40 & & 63 & 831 & - & & [97] \\
\hline & $\mathrm{C}_{10}$ & $1.5 \times 10^{5}$ & 1000 & 80 & - & $7.4 \times 10^{4}$ & 27 & 143 & - & & [95] \\
\hline 44 & $\mathrm{C}_{10}$ & $1.5 \times 10^{5}$ & 1000 & 80 & 5 & $1.2 \times 10^{5}$ & 17 & 71 & - & & [95] \\
\hline & $C_{10}$ & $1.5 \times 10^{5}$ & 1000 & 80 & 15 & $1.9 \times 10^{5}$ & 19 & 86 & - & & [95] \\
\hline 47 & $\mathrm{C}_{10}$ & $1.0 \times 10^{5}$ & 1000 & 40 & - & $1.0 \times 10^{5}$ & - & 702 & 296 & & [93] \\
\hline 48 & $\mathrm{C}_{10}$ & $2.6 \times 10^{5}$ & 600 & 80 & 6.9 & 611 & - & 11 & 211 & & [98] \\
\hline 51 & $C_{10}$ & $1.3 \times 10^{5}$ & 500 & 80 & 13.8 & $8.3 \times 10^{4}$ & - & 112 & 208 & & [98] \\
\hline & $\mathrm{C}_{10}$ & $1.0 \times 10^{5}$ & 1000 & 40 & - & $5.0 \times 10^{4}$ & - & 2460 & 344 & & [93] \\
\hline 52 & $\mathrm{C}_{10}$ & $2.6 \times 10^{5}$ & 200 & 100 & 13.8 & $8.9 \times 10^{4}$ & 21 & 116 & 214 & & [98] \\
\hline
\end{tabular}


Table 1. Cont.

\begin{tabular}{|c|c|c|c|c|c|c|c|c|c|c|}
\hline \multirow[t]{2}{*}{ Cat. } & \multirow[t]{2}{*}{ Mon. } & \multirow[t]{2}{*}[\mathrm{Mon}]{$/[\mathrm{Zr}]$} & \multirow[t]{2}{*}{$\begin{array}{l}{[\mathrm{Al}] /} \\
{[\mathrm{Zr}]}\end{array}$} & \multirow[t]{2}{*}{$\begin{array}{l}\mathrm{T}, \\
{ }^{\circ} \mathrm{C}\end{array}$} & \multirow{2}{*}{$\begin{array}{l}\mathrm{H}_{2} \\
\text { bar }\end{array}$} & \multirow[t]{2}{*}{ TOF, $h^{-1}$} & $D P_{\mathrm{n}}$ & $K V^{1001}$ & $\begin{array}{l}\text { Additional } \\
\text { Remarks }\end{array}$ & \multirow[t]{2}{*}{ Ref. } \\
\hline & & & & & & & \multicolumn{3}{|c|}{ or Oligomer Distribution for $D P_{n} 2,3,4,5$} & \\
\hline \multirow{6}{*}{53} & $\mathrm{C}_{10}$ & $2.6 \times 10^{5}$ & 250 & 100 & 6.9 & $2.6 \times 10^{5}$ & 19 & 94 & 213 & [98] \\
\hline & $\mathrm{C}_{10}$ & $2.6 \times 10^{5}$ & 250 & 90 & - & $5.6 \times 10^{4}$ & 133 & 1227 & - & [98] \\
\hline & $\mathrm{C}_{10}$ & $2.6 \times 10^{5}$ & 250 & 90 & 1.7 & $1.0 \times 10^{5}$ & 57 & 453 & - & [98] \\
\hline & $\mathrm{C}_{10}$ & $2.6 \times 10^{5}$ & 250 & 90 & 3.4 & $2.6 \times 10^{5}$ & 40 & 333 & - & [98] \\
\hline & $\mathrm{C}_{10}$ & $2.6 \times 10^{5}$ & 250 & 90 & 6.9 & $2.6 \times 10^{5}$ & 19 & 94 & - & [98] \\
\hline & $\mathrm{C}_{10}$ & $2.6 \times 10^{5}$ & 250 & 90 & 13.8 & $2.1 \times 10^{5}$ & 12 & 39 & 179 & [98] \\
\hline 56 & $\mathrm{C}_{10}$ & $4.2 \times 10^{5}$ & 260 & 80 & 13.8 & $1.4 \times 10^{5}$ & 21 & 25 & 183 & [98] \\
\hline \multirow{2}{*}{57} & $\mathrm{C}_{8}$ & $2.5 \times 10^{5}$ & 1000 & 65 & - & $1.4 \times 10^{5}$ & 23 & 139 & 192 & [99] \\
\hline & $\mathrm{C}_{8}$ & $2.5 \times 10^{5}$ & 1000 & 65 & 8 & $2.2 \times 10^{5}$ & 18 & 43 & 168 & [99] \\
\hline \multirow[t]{7}{*}{60} & $\mathrm{C}_{8}$ & $2.0 \times 10^{5}$ & 1000 & 25 & - & $1.3 \times 10^{5}$ & 83 & 1119 & - & [97] \\
\hline & $\mathrm{C}_{10}$ & $1.0 \times 10^{5}$ & 1000 & 40 & - & $1.0 \times 10^{5}$ & - & 2460 & 344 & [93] \\
\hline & $\mathrm{C}_{10}$ & $3.0 \times 10^{5}$ & 1000 & 70 & - & $2.4 \times 10^{5}$ & 64 & 635 & 282 & [93] \\
\hline & $\mathrm{C}_{10}$ & $3.0 \times 10^{5}$ & 1000 & 150 & - & $1.6 \times 10^{5}$ & - & 58 & 195 & [93] \\
\hline & $\mathrm{C}_{10}$ & $3.0 \times 10^{5}$ & 500 & 70 & - & $2.3 \times 10^{5}$ & - & 1134 & 307 & [93] \\
\hline & $\mathrm{C}_{10}$ & $3.0 \times 10^{5}$ & 250 & 70 & - & $1.7 \times 10^{5}$ & - & 1308 & 314 & [93] \\
\hline & $\mathrm{C}_{10}$ & $3.0 \times 10^{5}$ & 1000 & 70 & 0.5 & $1.5 \times 10^{5}$ & - & 1074 & 308 & [93] \\
\hline \multirow[t]{7}{*}{61} & $\mathrm{C}_{10}$ & $3.0 \times 10^{5}$ & 1000 & 70 & 1 & $2.1 \times 10^{5}$ & - & 863 & 296 & [93] \\
\hline & $\mathrm{C}_{10}$ & $3.0 \times 10^{5}$ & 1000 & 70 & 2 & $2.0 \times 10^{5}$ & - & 722 & 288 & [93] \\
\hline & $\mathrm{C}_{10}$ & $3.0 \times 10^{5}$ & 1000 & 70 & 13 & $2.4 \times 10^{5}$ & - & 512 & 271 & [93] \\
\hline & $\mathrm{C}_{6}$ & $3.0 \times 10^{5}$ & 1000 & 90 & - & $1.8 \times 10^{5}$ & - & 2862 & 251 & [93] \\
\hline & $\mathrm{C}_{8}$ & $3.0 \times 10^{5}$ & 1000 & 90 & - & $2.0 \times 10^{5}$ & - & 888 & 276 & [93] \\
\hline & $\mathrm{C}_{10}$ & $3.0 \times 10^{5}$ & 1000 & 90 & - & $1.6 \times 10^{5}$ & - & 515 & 272 & [93] \\
\hline & $\mathrm{C}_{12}$ & $3.0 \times 10^{5}$ & 1000 & 90 & - & $1.3 \times 10^{5}$ & - & 402 & 264 & [93] \\
\hline 62 & $\mathrm{C}_{10}$ & $7.0 \times 10^{5}$ & 1500 & 63 & 8 & $1.6 \times 10^{5}$ & 15 & 37 & 177 & [100] \\
\hline
\end{tabular}

${ }^{1}$ Kinematic viscosity at $100{ }^{\circ} \mathrm{C} .{ }^{2}$ Viscosity index. ${ }^{3}$ In $\%$ by weight, $D P_{\mathrm{n}}$ in the brackets, res.- residue, higher oligomers.

The degree of oligomerization, $D P_{\mathrm{n}}$, depends on the structure of the metallocene pre-catalyst, $\mathrm{Al}_{\mathrm{MAO}} / \mathrm{Zr}$ ratio, and reaction conditions. Unsubstituted zirconocene dichloride $\mathbf{1}$ and monosubstituted and disubstituted zirconocenes catalyzed the formation of the mixtures of lower oligomers. The presence of bulky alkyl or aryl substituents in the cyclopentadienyl rings resulted in decreasing activities of metallocenes and an increased content of higher oligomers; increasing the number of alkyl substituents entailed the same effect. The early results in the study of the oligomerization of $\alpha$-olefins in the presence of zirconocenes 1 and 8 at $\mathrm{Al}_{\mathrm{MAO}} / \mathrm{Zr} \sim 200$ [47] were complemented substantially by Nifant'ev et al. [91], who established that under these conditions, the products of side reactions (2-alkenes and alkanes) typically exceeded $10 \mathrm{wt} \%$. Therefore, bis-cyclopentadienyl complexes at high $\mathrm{Al}_{\mathrm{MAO}} / \mathrm{Zr}$ ratios have poor prospects for the oligomerization of $\alpha$-olefins.

The derivatives of substituted indenes and fluorenes demonstrated more promising catalytic properties. High yields of the oligomer fractions were obtained for metallocenes 53, 56 [98], and 61 [93]. For these complexes, the degree of oligomerization, $D P_{n}$, can be affected by the hydrogen pressure and reaction temperature with no reduction in the yield of the oligomer fraction.

In order to conclude this section on metallocene-catalyzed oligomerization at high $\mathrm{Al}_{\mathrm{MAO}} / \mathrm{Zr}$ ratios, it is important to note the publication of Jiang et al. [101], which was a thorough study of the microstructure of 1-butene/1-dodecene copolymers, obtained in the presence of $\left(\eta^{5}-\mathrm{C}_{5} \mathrm{Me}_{4} \mathrm{H}\right)_{2} \mathrm{ZrCl}_{2}$, activated by $100-500$ eq. of MAO. Detailed investigations of the kinetics and mechanisms of the formation of $\alpha$-olefin oligomers in the presence of $\left(\eta_{5}-\mathrm{C}_{5} \mathrm{H}_{4}-n-\mathrm{Bu}\right)_{2} \mathrm{ZrCl}_{2}$ [102] and $\left(\eta^{5}-\mathrm{C}_{5} \mathrm{H}_{5}\right)_{2} \mathrm{ZrCl}_{2}$ [103] after activation by $10^{2}-10^{3}$ eq. of MAO have also been of particular interest.

\subsection{Zirconocene-Catalyzed Oligomerization of $\alpha$-Olefins at Low $A l_{M A O} / Z r$ Ratios}

A systematic study of the zirconocene-catalyzed oligomerization of $\alpha$-olefins at low $\mathrm{Al}_{\mathrm{MAO}} / \mathrm{Zr}$ ratios was started in recent years. With the use of the method of the two-stage activation of $\mathrm{LZrCl}_{2}$ 
(with TIBA and then MAO, see Section 3.1), Nifant'ev et al. studied the catalytic performance of a wide range of zirconocenes [80,90-92]. The results of these studies are presented in Table 2.

Table 2. Zirconocene-catalyzed oligomerization of $\alpha$-olefins at low $\mathrm{Al} / \mathrm{Zr}$ ratios

\begin{tabular}{|c|c|c|c|c|c|c|c|c|c|}
\hline \multirow[t]{2}{*}{ Cat. } & \multirow[t]{2}{*}{ Mon. } & \multirow{2}{*}{$\begin{array}{c}\text { [Mon]/ } \\
{[\mathrm{Zr}]}\end{array}$} & \multirow{2}{*}{$\begin{array}{c}\mathbf{A l}_{\text {TIBA }} / \\
\mathbf{Z r}\end{array}$} & \multirow{2}{*}{$\underset{/ \mathrm{Zr}}{\mathrm{Al}_{\text {MAO }}}$} & \multirow{2}{*}{$\begin{array}{l}\mathrm{T}, \\
{ }^{\circ} \mathrm{C}\end{array}$} & \multirow{2}{*}{$\begin{array}{l}\mathrm{H}_{2} \\
\text { bar }\end{array}$} & \multirow{2}{*}{$\begin{array}{l}\text { Conv. } \\
\text { (h) }\end{array}$} & $\begin{array}{c}\text { Additional } \\
\text { Remarks }\end{array}$ & \multirow{2}{*}{ Ref. } \\
\hline & & & & & & & & or Oligomer Distribution & \\
\hline \multirow[t]{3}{*}{1} & $\mathrm{C}_{6}$ & $2 \times 10^{3}$ & 20 & 10 & 60 & - & $97(4)$ & $\%$ olig. 85 (2):12 (3-5), res. $1^{1}$ & [91] \\
\hline & $\mathrm{C}_{6}$ & $2 \times 10^{3}$ & 20 & 10 & 60 & - & $85(4)$ & \% olig. 35 (2):36 (3-5), res. 20 & [91] \\
\hline & $\mathrm{C}_{6}$ & $2 \times 10^{3}$ & 20 & 10 & 60 & 1 & $99(4)$ & $\%$ olig. 50 (2):31 (3-5), res. 11 & [91] \\
\hline \multirow[t]{3}{*}{8} & $\mathrm{C}_{8}$ & $2 \times 10^{3}$ & 20 & 10 & 60 & - & $83(4)$ & $\%$ olig. 40 (2):40 (3-5), res. 11 & [91] \\
\hline & $\mathrm{C}_{10}$ & $2 \times 10^{3}$ & 20 & 10 & 60 & - & $72(4)$ & \% olig. 38 (2):35 (3-5), res. 17 & [91] \\
\hline & $\mathrm{C}_{10}$ & $2 \times 10^{3}$ & 20 & 10 & 100 & - & $69(4)$ & $\%$ olig. 40 (2):36 (3-5), res. 10 & [91] \\
\hline 10 & $\mathrm{C}_{6}$ & $2 \times 10^{3}$ & 20 & 10 & 60 & - & $16(4)$ & \% olig. 40 (2):30 (3-5), res. 19 & [91] \\
\hline 18 & $\mathrm{C}_{6}$ & $2 \times 10^{3}$ & 20 & 10 & 60 & - & $92(4)$ & \% olig. 35 (2):31 (3-5), res. 31 & [91] \\
\hline 22 & $\mathrm{C}_{6}$ & $2 \times 10^{3}$ & 20 & 10 & 60 & - & $72(4)$ & \% olig. $8(2): 34(3-5)$, res. 55 & [91] \\
\hline 26 & $\mathrm{C}_{6}$ & $4 \times 10^{3}$ & 80 & 40 & 60 & - & $90(4)$ & 38 & [92] \\
\hline 30 & $\mathrm{C}_{6}$ & $2 \times 10^{3}$ & 20 & 10 & 60 & - & $89(4)$ & 44 & [91] \\
\hline \multirow[t]{2}{*}{31} & $\mathrm{C}_{6}$ & $4 \times 10^{3}$ & 80 & 40 & 60 & - & $75(4)$ & 190 & [92] \\
\hline & $\mathrm{C}_{6}$ & $2 \times 10^{3}$ & 20 & 10 & 60 & - & $98(4)$ & \% olig. 41 (2):28 (3-5), res. 24 & [91] \\
\hline \multirow{3}{*}{35} & $\mathrm{C}_{8}$ & $2 \times \cdot 10^{3}$ & 20 & 10 & 60 & - & $98(4)$ & $\%$ olig. 36 (2):32 (3-5), res. 29 & [91] \\
\hline & $\mathrm{C}_{10}$ & $2 \times 10^{3}$ & 20 & 10 & 60 & - & $98(4)$ & \% olig. 40 (2):33 (3-5), res. 35 & [91] \\
\hline & $\mathrm{C}_{10}$ & $2 \times 10^{3}$ & 20 & 10 & 100 & - & $98(4)$ & \% olig. 41 (2):34 (3-5), res. 19 & [91] \\
\hline 36 & $\mathrm{C}_{6}$ & $2 \cdot \times 10^{3}$ & 20 & 10 & 60 & - & $98(4)$ & $\%$ olig. 40 (2):30 (3-5), res. 11 & [90] \\
\hline 41 & $\mathrm{C}_{6}$ & $2 \times 10^{3}$ & 20 & 10 & 60 & - & $95(4)$ & \% olig. $22(2): 32(3-5)$, res. 39 & [91] \\
\hline 42 & $\mathrm{C}_{6}$ & $2 \times 10^{3}$ & 20 & 10 & 60 & - & $94(4)$ & \% olig. 20 (2):31 (3-5), res. 40 & [91] \\
\hline \multirow{2}{*}{45} & $\mathrm{C}_{6}$ & $2 \cdot \times 10^{3}$ & 20 & 10 & 60 & - & $88(4)$ & 25 & [91] \\
\hline & $\mathrm{C}_{6}$ & $4 \times 10^{3}$ & 80 & 40 & 60 & - & $76(4)$ & 25 & [92] \\
\hline 46 & $\mathrm{C}_{6}$ & $2 \times 10^{3}$ & 20 & 10 & 60 & - & $94(4)$ & 108 & [91] \\
\hline \multirow{2}{*}{47} & $\mathrm{C}_{6}$ & $2 \times 10^{3}$ & 20 & 10 & 60 & - & $92(4)$ & 37 & [91] \\
\hline & $\mathrm{C}_{6}$ & $4 \times 10^{3}$ & 80 & 40 & 60 & - & $94(4)$ & 35 & [92] \\
\hline 50 & $\mathrm{C}_{6}$ & $4 \times 10^{3}$ & 80 & 40 & 60 & - & $95(4)$ & 108 & [92] \\
\hline \multirow[t]{6}{*}{55} & $\mathrm{C}_{6}$ & $4 \times 10^{3}$ & 80 & 40 & 60 & - & $95(4)$ & 68 & [92] \\
\hline & $\mathrm{C}_{6}$ & $2 \times 10^{3}$ & 20 & 10 & 60 & - & $100(4)$ & $\%$ olig. $9(2): 17(3-5)$, res. 72 & [91] \\
\hline & $\mathrm{C}_{6}$ & $2 \times 10^{3}$ & 20 & 10 & 60 & 1 & $100(4)$ & \% olig. 13 (2):24 (3-5), res. 57 & [91] \\
\hline & $\mathrm{C}_{8}$ & $2 \times 10^{3}$ & 20 & 10 & 60 & - & $100(4)$ & \% olig. 23 (2):30 (3-5), res. 45 & [91] \\
\hline & $\mathrm{C}_{8}$ & $2 \times 10^{3}$ & 20 & 10 & 80 & - & $100(4)$ & \% olig. 28 (2):26 (3-5), res. 44 & [91] \\
\hline & $\mathrm{C}_{8}$ & $2 \times 10^{3}$ & 20 & 10 & 100 & - & $100(4)$ & \% olig. 37 (2):34 (3-5), res. 27 & [91] \\
\hline \multirow[t]{6}{*}{59} & $\mathrm{C}_{8}$ & $2 \times 10^{3}$ & 20 & 10 & 120 & - & $100(4)$ & \% olig. 44 (2):32 (3-5), res. 21 & [91] \\
\hline & $\mathrm{C}_{8}$ & $2 \times 10^{3}$ & 20 & 10 & 100 & 1 & $100(4)$ & \% olig. 47 (2):34 (3-5), res. 8 & [91] \\
\hline & $\mathrm{C}_{8}$ & $2 \times 10^{3}$ & 20 & 10 & 120 & 1 & $100(4)$ & $\%$ olig. 51 (2):40 (3-5), res. 10 & [91] \\
\hline & $\mathrm{C}_{10}$ & $2 \times 10^{3}$ & 20 & 10 & 100 & - & $93(4)$ & \% olig. $44(2): 45(3-5)$, res. 7 & [91] \\
\hline & $\mathrm{C}_{10}$ & $2 \times 10^{3}$ & 20 & 10 & 100 & 1 & $99(4)$ & \% olig. 45 (2):41 (3-5), res. 6 & [91] \\
\hline & $\mathrm{C}_{10}$ & $2 \times 10^{3}$ & 20 & 10 & 120 & 1 & $99(4)$ & \% olig. 51 (2):33 (3-5), res. 10 & [91] \\
\hline
\end{tabular}

During the research, the side reactions of the isomerization and reduction of the starting $\alpha$-olefins were studied. In was established that in some cases, the content of 2-alkenes in the reaction products can reach values of $20 \%$ and higher (for example, for zirconocene 36 [90]). The formation of 2-alkenes is a greatly underestimated problem of the metallocene-catalyzed oligomerization of $\alpha$-olefins; the isomerization of the starting monomers to inert internal olefins seriously diminishes the yields of oligomers, thus generally devaluing the method of metallocene-catalyzed oligomerization in the production of polyolefin oils and lubricants.

The feasibility of the ligand design is a substantial advantage of the zirconocene catalysis; the inherently high stability of the bis $\left(\eta^{5}\right.$-cyclopentadienyl $) \mathrm{Zr}$ fragment allows for a fruitful search of the catalyst without the drawbacks of the unnecessary wasting of the starting $\alpha$-olefins. 
Figure 2 illustrates this idea; activation by 10 eq. of modified methylalumoxane MMAO-12, bis-cyclopentadienyl complex 8 was clearly inferior to that by ansa-zirconocene 35 in terms of catalytic activity, but both zirconocenes 8 and 35 were less active and selective than zirconocene 59 [91]. Using 59, Nifant'ev et al. separated oligomers of 1-hexene, 1-octene, and 1-decene for $D P_{n}=2-5$ and proved the homogeneity of their structure [91]; similar results were reported later by Mi et al., who used the less active zirconium complexes 1, 2, 10, and 50 [104]. The complex 59 is a representative of metallocenes containing heterocycle-fused $\eta^{5}$-cyclopentadienyl fragments, "heterocenes". In the early 2000s, such complexes were extensively studied by the chemists of the Basell Polyolefins and Exxon Mobil companies in close cooperation with M.V. Lomonosov Moscow University in the polymerization of ethylene and propylene [105-108]. To date, heterocenes are considered as the most promising single-site oligomerization catalysts (see Sections 4.2 and 5).

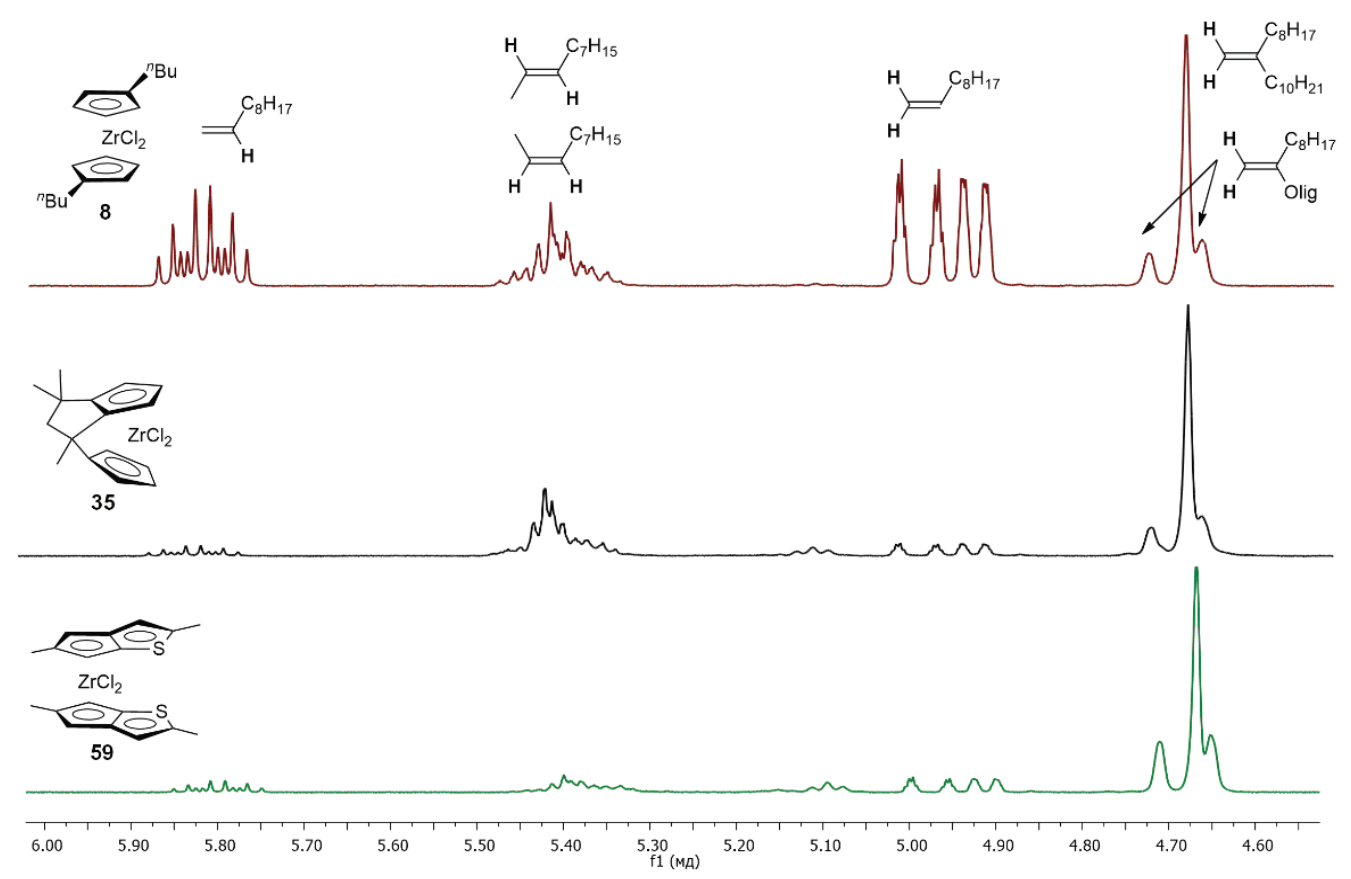

Figure 2. Fragments of ${ }^{1} \mathrm{H}$ NMR spectra of the reaction mixtures of 1-decene oligomerization ( $2 \mathrm{~h}$, $100{ }^{\circ} \mathrm{C}$ ), catalyzed by zirconocenes 8,35 , and 59 , activated by 10 eq. of MMAO-12. Reprinted with permission from [91]. Copyright (2018) Elsevier B.V.

\subsection{Zirconocene-Catalyzed Oligomerization of $\alpha$-Olefins, Activated by Perfluoroaryl Borates}

To avoid the use of a large excess of organoaluminium in the activation of metallocenes, MAO can be replaced with perfluoroaryl borates. This method, proposed by Marks et al. [109-111], has been successfully used in the oligomerization of $\alpha$-olefins (Table 3).

The activation by perfluoroalkyl borates, apparently, resulted in the formation of more electrophilic separate ion pairs, which caused the possibility of side processes unusual for zirconocene/MAO catalysts. The ${ }^{1} \mathrm{H}$ NMR analysis of the end-groups of 1-decene oligomers, obtained in the presence of zirconocenes 32-34, clearly demonstrated the product $\beta$-hydride's elimination from the secondary $\mathrm{Zr}$-alkyl complexes [112]. The formation of such complexes does not affect the carbon skeleton of the oligomers; however, it inevitably results in the slowing down of the oligomerization. A substantially more important process-namely, the $\beta$-elimination of the $n$-hexyl fragment (Scheme 5 , reaction pathway C)-was detected in the oligomerization of 1-octene catalyzed by bis(indenyl) hafnium complex 54 [73]. The products of this side reaction represent allyl-terminated oligooctenes that are able to react with the formation of long-chain branched polymers. 
Table 3. Zirconocene-catalyzed oligomerization of $\alpha$-olefins, with activation by perfluoroaryl borates.

\begin{tabular}{|c|c|c|c|c|c|c|c|c|c|c|c|}
\hline \multirow[b]{2}{*}{ Cat. } & \multirow[b]{2}{*}{ Mon. } & \multirow[b]{2}{*}{$\begin{array}{l}{[\mathrm{Mon}]} \\
/[\mathrm{Zr}]\end{array}$} & \multirow[b]{2}{*}{$\mathbf{A l} / \mathbf{Z r}$} & \multirow[b]{2}{*}{${ }^{\mathrm{T}}{ }^{\circ} \mathrm{C}$} & \multirow[b]{2}{*}{$\begin{array}{l}\mathrm{H}_{2} \\
\text { bar }\end{array}$} & \multirow[b]{2}{*}{ TOF, $h^{-1}$} & $D P_{\mathrm{n}}$ & $K V^{100}$ & VI & \multirow{2}{*}{$\begin{array}{c}\mathrm{AlR}_{3} \\
\text { Additional } \\
\text { Remarks }\end{array}$} & \multirow[b]{2}{*}{ Ref. } \\
\hline & & & & & & & \multicolumn{3}{|c|}{$\begin{array}{l}\text { or Oligomer Distribution for } \\
\qquad D P_{\mathrm{n}} 2,3,4,5+\end{array}$} & & \\
\hline \multirow{3}{*}{$11, X=M e$} & $\mathrm{C}_{10}$ & $5.2 \times 10^{5}$ & 50 & 100 & - & $1.0 \cdot \times 10^{3}$ & 27 & 103 & - & $\mathrm{Al}(n-\mathrm{Oct})_{3}, \mathrm{NB}^{1}$ & [113] \\
\hline & $\mathrm{C}_{10}$ & $5.2 \cdot \times 10^{5}$ & 50 & 100 & 3 & $3.6 \cdot \times 10^{5}$ & 23 & 11.3 & - & $\mathrm{Al}(n-\mathrm{Oct})_{3}, \mathrm{NB}$ & [113] \\
\hline & $C_{10}$ & $5.2 \times 10^{5}$ & 50 & 100 & - & $5.2 \cdot \times 10^{4}$ & 10.6 & 4.5 & _- & $\mathrm{Al}(n-\mathrm{Oct})_{3}, \mathrm{NB}$ & [113] \\
\hline \multirow{4}{*}{$16, X=M e$} & $\mathrm{C}_{10}$ & $5.2 \cdot \times 10^{5}$ & 50 & 120 & - & $3.1 \cdot \times 10^{4}$ & 8.8 & 3.4 & - & $\mathrm{Al}(n-\mathrm{Oct})_{3}, \mathrm{NB}$ & [113] \\
\hline & $\mathrm{C}_{10}$ & $5.2 \cdot \times 10^{5}$ & 50 & 100 & 3.7 & $7.8 \times 10^{4}$ & 9.5 & 3.7 & _- & $\mathrm{Al}(n-\mathrm{Oct})_{3}, \mathrm{NB}$ & [113] \\
\hline & $\mathrm{C}_{10}$ & $5.2 \cdot \times 10^{5}$ & 50 & 120 & 3 & $8.0 \times 10^{4}$ & 8.3 & 3.1 & - & $\mathrm{Al}(n-\mathrm{Oct})_{3}, \mathrm{NB}$ & [113] \\
\hline & $\mathrm{C}_{10}$ & $5.2 \cdot \times 10^{5}$ & 50 & 100 & - & $8.4 \cdot \times 10^{3}$ & 18 & 10.2 & _- & $\mathrm{Al}(n-\mathrm{Oct})_{3}, \mathrm{NB}$ & [113] \\
\hline \multirow[t]{3}{*}{$17, X=M e$} & $\mathrm{C}_{10}$ & $5.2 \times 10^{5}$ & 50 & 120 & - & $1.2 \cdot \times 10^{4}$ & 11 & 4.9 & - & $\mathrm{Al}(n-\mathrm{Oct})_{3}, \mathrm{NB}$ & [113] \\
\hline & $\mathrm{C}_{10}$ & $5.2 \cdot \times 10^{5}$ & 50 & 120 & 3 & $5.9 \cdot \times 10^{4}$ & 10.6 & 4.5 & - & $\mathrm{Al}(n-\mathrm{Oct})_{3}, \mathrm{NB}$ & [113] \\
\hline & $\mathrm{C}_{10}$ & $5.2 \cdot \times 10^{5}$ & 50 & 100 & - & $6.3 \times 10^{4}$ & 9.3 & 4.0 & - & $\mathrm{Al}(n-\mathrm{Oct})_{3}, \mathrm{NB}$ & [113] \\
\hline \multirow{3}{*}{8} & $\mathrm{C}_{10}$ & $5.2 \cdot \times 10^{5}$ & 50 & 120 & - & $3.9 \times 10^{4}$ & 7.9 & 3.1 & - & $\mathrm{Al}(n-\mathrm{Oct})_{3}, \mathrm{NB}$ & [113] \\
\hline & $\mathrm{C}_{10}$ & $5.2 \cdot \times 10^{5}$ & 50 & 100 & 3 & $7.4 \cdot \times 10^{4}$ & 9.0 & 3.8 & - & $\mathrm{Al}(n-\mathrm{Oct})_{3}, \mathrm{NB}$ & [113] \\
\hline & $\mathrm{C}_{10}$ & $5.2 \times 10^{5}$ & 50 & 120 & 3 & $8.8 \times 10^{4}$ & 7.9 & 3.1 & - & $\mathrm{Al}(n-\mathrm{Oct})_{3}, \mathrm{NB}$ & [113] \\
\hline & $\mathrm{C}_{10}$ & $5.2 \cdot \times 10^{5}$ & 50 & 100 & 3 & $8.7 \times \times 10^{4}$ & 7.9 & 3.0 & - & $\mathrm{Al}(n-\mathrm{Oct})_{3}, \mathrm{NB}$ & [113] \\
\hline 20 & $\mathrm{C}_{10}$ & $5.2 \times 10^{5}$ & 50 & 120 & 3 & $8.8 \cdot \times 10^{4}$ & 7.5 & 2.8 & _- & $\mathrm{Al}(n-\mathrm{Oct})_{3}, \mathrm{NB}$ & [113] \\
\hline & $\mathrm{C}_{10}$ & $8.5 \cdot \times 10^{4}$ & 4 & 50 & - & $1.3 \cdot \times 10^{4}$ & - & 96 & 199 & $\mathrm{Al}(n-\mathrm{Oct})_{3}, \mathrm{NB}$ & [114] \\
\hline & $\mathrm{C}_{10}$ & $8.5 \cdot \times 10^{4}$ & 4 & 60 & 1 & $1.6 \cdot \times 10^{4}$ & - & 44 & 180 & $\mathrm{Al}(n-\mathrm{Oct})_{3}, \mathrm{NB}$ & [114] \\
\hline & $\mathrm{C}_{10}$ & $8.5 \cdot \times 10^{4}$ & 4 & 70 & 1 & $1.8 \times 10^{4}$ & - & 20 & 179 & $\mathrm{Al}(n-\mathrm{Oct})_{3}, \mathrm{NB}$ & [114] \\
\hline & $C_{10}$ & $8.5 \times 10^{4}$ & 4 & 80 & 1 & $2.0 \cdot \times 10^{4}$ & - & 11.0 & 163 & $\mathrm{Al}(n-\mathrm{Oct})_{3}, \mathrm{NB}$ & [114] \\
\hline $21, X=M e$ & $\mathrm{C}_{10}$ & $8.5 \cdot \times 10^{4}$ & 4 & 100 & 1 & $1.7 \cdot \times 10^{4}$ & - & 8.0 & 161 & $\mathrm{Al}(n-\mathrm{Oct})_{3}, \mathrm{NB}$ & [114] \\
\hline & $\mathrm{C}_{10}$ & $8.5 \cdot \times 10^{4}$ & 4 & 120 & 1 & $2.1 \times 10^{4}$ & - & 5.0 & 151 & $\mathrm{Al}(n-\mathrm{Oct})_{3}, \mathrm{NB}$ & [114] \\
\hline & $\mathrm{C}_{10}$ & $5.2 \cdot \times 10^{5}$ & 50 & 80 & - & $2.2 \cdot \times 10^{4}$ & 13 & 5.9 & - & $\mathrm{Al}(n-\mathrm{Oct})_{3}, \mathrm{NB}$ & [113] \\
\hline & $\mathrm{C}_{10}$ & $5.2 \cdot \times 10^{5}$ & 50 & 100 & - & $3.3 \times \cdot 10^{4}$ & 10 & 4.1 & 148 & $\mathrm{Al}(n-\mathrm{Oct})_{3}, \mathrm{NB}$ & [113] \\
\hline & $\mathrm{C}_{10}$ & $5.2 \cdot \times 10^{5}$ & 50 & 120 & - & $3.3 \cdot \times 10^{4}$ & 8.5 & 3.3 & - & $\mathrm{Al}(n-\mathrm{Oct})_{3}, \mathrm{NB}$ & [113] \\
\hline & $C_{10}$ & $5.2 \times 10^{5}$ & 50 & 100 & 3 & $1.2 \times 10^{5}$ & 8.6 & 3.3 & _- & $\mathrm{Al}(n-\mathrm{Oct})_{3}, \mathrm{NB}$ & [113] \\
\hline 26 & $\mathrm{C}_{10}$ & $1.2 \cdot \times 10^{5}$ & 100 & 110 & - & - & - & 3.9 & 144 & TIBA, NB & [115] \\
\hline & $\mathrm{C}_{10}$ & $5.2 \cdot \times 10^{5}$ & 50 & 100 & - & $7.2 \times 10^{3}$ & 10 & 4.1 & - & $\mathrm{Al}(n-\mathrm{Oct})_{3}, \mathrm{NB}$ & [113] \\
\hline $97 \mathrm{Y}$ & $C_{10}$ & $5.2 \times 10^{5}$ & 50 & 120 & _- & $1.8 \cdot \times 10^{4}$ & 8.3 & 3.1 & _- & $\mathrm{Al}(n-\mathrm{Oct})_{3}, \mathrm{NB}$ & [113] \\
\hline $27, X=M e$ & $\mathrm{C}_{10}$ & $5.2 \cdot \times 10^{5}$ & 50 & 100 & 3 & $1.1 \cdot \times 10^{5}$ & 9.3 & 3.6 & - & $\mathrm{Al}(n-\mathrm{Oct})_{3}, \mathrm{NB}$ & [113] \\
\hline & $\mathrm{C}_{10}$ & $5.2 \times 10^{5}$ & 50 & 120 & 3 & $3.1 \times 10^{4}$ & 8.0 & 3.0 & - & $\mathrm{Al}(n-\mathrm{Oct})_{3}, \mathrm{NB}$ & [113] \\
\hline & $\mathrm{C}_{10}$ & $5.2 \cdot \times 10^{5}$ & 50 & 100 & 3 & $2.0 \times 10^{4}$ & 7.6 & 2.8 & - & $\mathrm{Al}(n-\mathrm{Oct})_{3}, \mathrm{NB}$ & [113] \\
\hline,$X=M e$ & $\mathrm{C}_{10}$ & $5.2 \cdot \times 10^{5}$ & 50 & 120 & 3 & $1.4 \times 10^{4}$ & 7.9 & 3.2 & _- & $\mathrm{Al}(n-\mathrm{Oct})_{3}, \mathrm{NB}$ & [113] \\
\hline 32 & $\mathrm{C}_{10}$ & $2.6 \cdot \times 10^{5}$ & 200 & 120 & - & $4.7 \times \times 10^{4}$ & & $/ 7.0 / 4.0 / 2$ & +64 & TIBA, $\mathrm{CB}^{2}$ & [112] \\
\hline & $\mathrm{C}_{8}$ & $2.6 \cdot \times 10^{5}$ & 200 & 120 & - & $1.9 \cdot \times 10^{5}$ & & $.1 / 14.1 / 11$ & 33.4 & TIBA, CB & [112] \\
\hline 33 & $\mathrm{C}_{10}$ & $2.6 \cdot \times 10^{5}$ & 200 & 120 & _- & $1.5 \cdot \times 10^{5}$ & & $3 / 14.3 / 11$ & 34.7 & TIBA, CB & [112] \\
\hline 34 & $\mathrm{C}_{10}$ & $2.6 \cdot \times 10^{5}$ & 200 & 120 & - & $9.1 \times 10^{4}$ & & $0 / 18.4 / 13$ & 24.6 & TIBA, CB & [112] \\
\hline 30 & $\mathrm{C}_{10}$ & $1.5 \times 10^{5}$ & 200 & 120 & + & $1.2 \cdot \times 10^{5}$ & 6.6 & 5.5 & 164 & TIBA, NB & [95] \\
\hline 38 & $\mathrm{C}_{10}$ & $1.5 \cdot \times 10^{5}$ & 200 & 140 & + & $1.2 \times 10^{5}$ & 4.4 & 2.6 & 140 & TIBA, NB & [95] \\
\hline & $\mathrm{C}_{10}$ & $2.0 \times 10^{5}$ & 20 & 120 & - & $\sim 2 \times 10^{5}$ & $\sim 3$ & $\begin{array}{l}\text { C } 303.4 \\
\text { C40 9.3 }\end{array}$ & 128158 & $\mathrm{Al}(n-\mathrm{Oct})_{3}, \mathrm{NB}$ & [52, \\
\hline $39, X=M e$ & $\mathrm{C}_{10}$ & $8.9 \times 10^{4}$ & 0.01 & 60 & - & $7.3 \times 10^{4}$ & 32 & 169 & 225 & $\mathrm{Al}(n-\mathrm{Oct})_{3}, \mathrm{NB}$ & [116] \\
\hline & $\mathrm{C}_{10}$ & $8.9 \cdot \times 10^{4}$ & 0.01 & 80 & - & $8.4 \times 10^{4}$ & 14 & 26.5 & 169 & $\mathrm{Al}(n-\mathrm{Oct})_{3}, \mathrm{NB}$ & [116] \\
\hline & $\mathrm{C}_{10}$ & $8.9 \cdot \times 10^{4}$ & 0.01 & 100 & - & $8.8 \cdot \times 10^{4}$ & 9.3 & 11.2 & 150 & $\mathrm{Al}(n-\mathrm{Oct})_{3}, \mathrm{NB}$ & [116] \\
\hline & $C_{10}$ & $3.6 \cdot \times 10^{5}$ & $>100$ & 70 & _- & $3.1 \cdot \times 10^{5}$ & - & 18.5 & 164 & TIBA, CB & [117] \\
\hline 40 & $\mathrm{C}_{10}$ & $3.6 \cdot \times 10^{5}$ & $>100$ & 100 & - & $2.0 \times 10^{5}$ & - & 8.9 & 153 & TIBA, NB & [117] \\
\hline & $C_{10}$ & $3.6 \cdot \times 10^{5}$ & $>100$ & 100 & - & $2.5 \times 10^{5}$ & - & 14.6 & 158 & TIBA, CB & [117] \\
\hline & $\mathrm{C}_{10}$ & $3.5 \cdot \times 10^{5}$ & $>100$ & 50 & - & $1.5 \times 10^{5}$ & 226 & 3715 & 379 & TIBA, NB & [117] \\
\hline 48 & $\mathrm{C}_{10}$ & $3.5 \cdot \times 10^{5}$ & $>100$ & 84 & - & $2.9 \cdot \times 10^{5}$ & 80 & 724 & 291 & TIBA, NB & [117] \\
\hline & $\mathrm{C}_{10}$ & $3.5 \cdot \times 10^{5}$ & $>100$ & 100 & - & $2.3 \cdot \times 10^{5}$ & 64 & 363 & 250 & TIBA, NB & [117] \\
\hline 50 & $C_{10}$ & $2-4 \times 10^{5}$ & $20-200$ & $60-10$ & 0 -10 & $1.0 \cdot \times 10^{5}$ & - & $253-586$ & $247-281$ & TIBA, NB & [118] \\
\hline & $\mathrm{C}_{8} / \mathrm{C}_{12}$ & $1.0 \cdot \times 10^{5}$ & 10 & 100 & 0.5 & $\sim 1 \cdot \times 10^{5}$ & $15-35$ & $39-140$ & $175-212$ & TIBA, NB & [119] \\
\hline 58 & $\mathrm{C}_{8} / \mathrm{C}_{10} / \mathrm{C}_{1}$ & $21.0 \times 10^{5}$ & 10 & 100 & 0.5 & $\sim 1 \cdot \times 10^{5}$ & $15-30$ & $40-120$ & 179-205 & TIBA, NB & [120] \\
\hline & $\mathrm{C}_{8} / \mathrm{C}_{10}$ & $1.0 \cdot \times 10^{5}$ & 10 & 100 & 0.5 & $\sim 1 \cdot \times 10^{5}$ & $19-32$ & $45-109$ & $175-204$ & TIBA, NB & [121] \\
\hline & $\mathrm{C}_{10}$ & $4.4 \cdot \times 10^{5}$ & 10 & 80 & 2 & $3.6 \cdot \times 10^{5}$ & - & 834 & 304 & TIBA, NB & [122] \\
\hline 61 & $\mathrm{C}_{10}$ & $4.4 \times 10^{5}$ & 10 & 105 & 2 & $2.4 \times \times 10^{5}$ & 76 & 622 & 289 & TIBA, NB & [123, \\
\hline & $\mathrm{C}_{10}$ & $4.4 \times 10^{5}$ & 10 & 110 & 2 & $3.2 \times 10^{5}$ & 71 & 558 & 280 & TIBA, NB & $\begin{array}{l}124] \\
{[122]}\end{array}$ \\
\hline & $\mathrm{C}_{10}$ & $4.4 \times 10^{5}$ & 10 & 120 & 2 & $2.4 \cdot \times 10^{5}$ & 63 & 434 & 270 & TIBA, NB & [122] \\
\hline & $\mathrm{C}_{10}$ & $4.4 \cdot \times 10^{5}$ & 10 & 130 & 2 & $2.4 \times 10^{5}$ & 57 & 377 & 266 & TIBA, NB & [122] \\
\hline
\end{tabular}

Experiments on the oligomerization of 1-decene in the presence of the $47-\mathrm{Me} /[\mathrm{PhNHMe} 2]\left[\mathrm{B}\left(\mathrm{C}_{6} \mathrm{~F}_{5}\right)_{4}\right]$ catalytic system, performed by the chemists of Total company [125], deserve special notice. The content of the $\mathrm{C}_{30}$ fraction in the reaction products reached a value of $70 \%$, comparing favorably with those in $\mathrm{BF}_{3}$-catalyzed oligomerization. 
In addition to MAO and perfluoroaryl borates, fluorinated aluminosilicate, in combination with TIBA, was successfully used in the zirconocene-catalyzed oligomerization of $\alpha$-olefins [97] (Table 4).

Table 4. Oligomerization of 1-octene, catalyzed by zirconium complexes, activated by triisobutylalumnium (TIBA) and fluorinated aluminosilicate [97].

\begin{tabular}{cccccc}
\hline Cat. & {$[\mathbf{M o n}] /[\mathbf{Z r}]$} & $\mathbf{T}^{\circ}{ }^{\circ} \mathbf{C}$ & $\mathbf{T O F} \mathbf{h}^{\mathbf{1}}$ & $\mathbf{K V}^{\mathbf{1 0 0}}$ & $\boldsymbol{V I}$ \\
\hline $\mathbf{1}$ & $1.1 \times 10^{5}$ & 90 & $3.1 \times 10^{3}$ & 8.9 & 211 \\
$\mathbf{8}$ & $5.6 \times 10^{5}$ & 105 & $4.1 \times 10^{4}$ & 45 & 175 \\
$\mathbf{9}$ & $3.1 \times 10^{5}$ & 110 & $3.4 \times 10^{4}$ & 62 & 186 \\
$\mathbf{1 5}$ & $1.5 \times 10^{6}$ & 70 & $1.3 \times 10^{5}$ & 130 & 222 \\
$\mathbf{2 9}$ & $5.5 \times 10^{5}$ & 90 & $2.5 \times 10^{4}$ & 8.3 & 157 \\
$\mathbf{4 2}$ & $4.3 \times 10^{5}$ & 120 & $3.1 \times 10^{4}$ & 159 & 214 \\
$\mathbf{4 3}$ & $4.4 \times 10^{5}$ & 120 & $3.6 \times 10^{4}$ & 132 & 200 \\
$\mathbf{4 5}$ & $1.1 \times 10^{5}$ & 90 & $9.0 \times 10^{3}$ & 23 & 169 \\
$\mathbf{4 7}$ & $4.2 \times 10^{5}$ & 115 & $4.3 \times 10^{4}$ & 136 & 210 \\
$\mathbf{4 9}$ & $1.0 \times 10^{5}$ & 100 & $5.2 \times 10^{3}$ & 10.3 & 194 \\
\hline
\end{tabular}

\subsection{Post-Metallocene Catalysts in the Oligomerization of $\alpha$-Olefins}

Post-metallocene catalysts that have been intensively and fruitfully studied in the polymerization and oligomerization of ethylene and propylene [126-134] were of limited use in the oligomerization of higher $\alpha$-olefins. The Group 8 metal complexes 70 [135] and 71 [136] (Scheme 6) were inferior to zirconocenes in terms of catalytic activity and, therefore, are of no interest for practical applications. The Group 4 post-metallocenes have greater potential in selective oligomerization. The carbene zirconium complex $\mathbf{7 2}$ with bulky phenolate fragments (Scheme 7) in the presence of $\left[\mathrm{Ph}_{3} \mathrm{C}\right]\left[\mathrm{B}\left(\mathrm{C}_{6} \mathrm{~F}_{5}\right)_{4}\right]$ demonstrated moderate activity in the non-selective oligomerization of 1-hexene; however, activation by $[\mathrm{PhNHMe} 2]\left[\mathrm{B}\left(\mathrm{C}_{6} \mathrm{~F}_{5}\right)_{4}\right]$ resulted in the formation of a trimerization product with $\sim 77 \%$ yield [137]. At the same time, the benzimidazole analog 73 catalyzed non-selective oligomerization regardless of the type of activator [138]. Zirconium complexes with [OSSO]-type ligands 74, 75 [139], and 76 [140] (Scheme 6) in the presence of dried modified methylaluminoxane (dMMAO) catalyzed the formation of lower 1-hexene oligomers with excellent vinylidene selectivity. Apparently, the mechanism of the oligomerization catalyzed by $\mathbf{7 2 - 7 6}$ is similar to the mechanism of zirconocene-catalyzed oligomerization.

The metallacyclic mechanism of the coordination oligomerization of $\alpha$-olefins (Scheme 7a) $[130,141]$ is highly attractive regarding the selectivity of the formation of the trimer fraction. This mechanism is considered proven for chromium (III) complexes 77-82 of tridentate cyclic ligands (Scheme 7b) [142-145]. In the presence of 100 eq. of MAO, the complexes 77 and 78 catalyzed the trimerization of 1-hexene with high selectivity; dimers and tetramers were found in less than $1 \%$, and TONs $\sim 10^{3}$ were detected at room temperature [142]. The structures and ratios of the major isomers in the $\mathrm{C}_{18}$ fraction are presented in Scheme 7c. One year later [143], Wasserscheid et al. studied the trimerization of 1-decene and 1-dodecene, catalyzed by a series of the complexes 79-82 with different substituents at $\mathrm{N}$ atoms; the complex 80 with 2-ethylhexyl substituents demonstrated the best catalytic performance in terms of trimerization selectivity and productivity. The impact of the steric bulk of the alkyl substituent $\mathrm{R}^{\prime}$ on trimerization selectivity was studied in 2016 by Cohon and Köhn [144] for complexes 83-86 (Scheme $7 b$ ). The ratios of the products of trimerization of 1-hexene are presented in Table 5. 


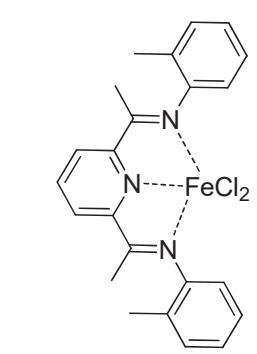

70

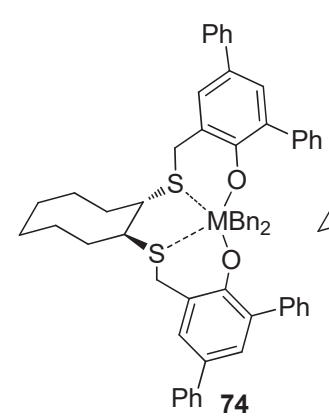

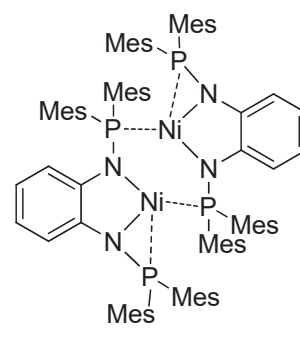

71

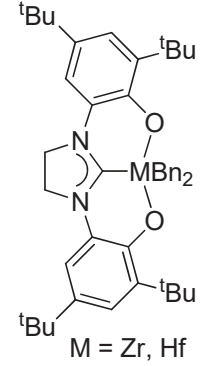

72<smiles>CC12Oc3c(Br)cc(Br)cc3N3c4ccccc4N(c4cc(Br)cc(Br)c4O1)C32C</smiles>

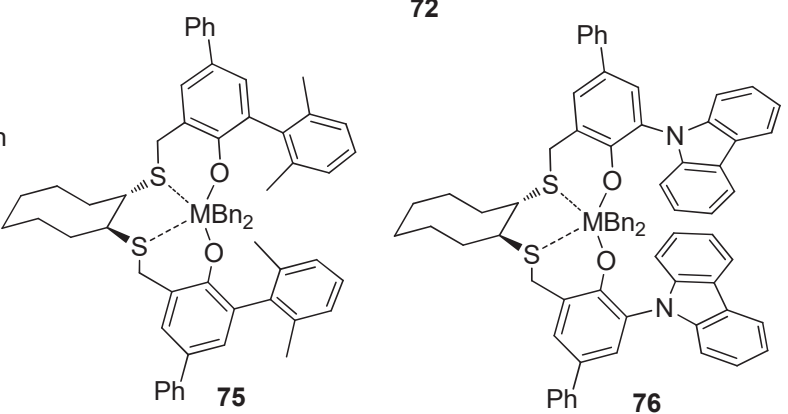

Scheme 6. Post-metallocene pre-catalysts studied in the non-selective coordination oligomerization of $\alpha$-olefins.

(a)

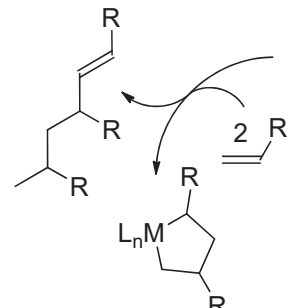<smiles>[R]C=CC([R])CC([R])CNCCCCCCCC([R])CC([R])CC</smiles><smiles>C=CC(C)CC</smiles>

$77 . R=R^{\prime}=n-C_{1} H_{13}$

(b)<smiles>[R]N1C2(Cl)CN3CN2C(Cl)(Cl)C31Cl</smiles>

$78, \mathrm{R}=\mathrm{R}^{\prime}=n-\mathrm{C}_{12} \mathrm{H}_{25}$

$79, R=R^{\prime}=1,5$-dimethylhexyl

$80, R=R^{\prime}=2$-ethylhexyl

81, $R=R^{\prime}=1,1$-dimethylundecyl

82, $R=R^{\prime}=C H(M e) P h$

83, R $=n-\mathrm{C}_{5} \mathrm{H}_{11}, \mathrm{R}^{\prime}=\mathrm{CH}_{2} \mathrm{CH}_{2} \mathrm{CH}(n-\mathrm{Pent})_{2}$

$84, \mathrm{R}=n-\mathrm{C}_{5} \mathrm{H}_{11}, \mathrm{R}^{\prime}=\mathrm{CH}_{2} \mathrm{CH}_{2} \mathrm{CCl}(n-\mathrm{Pent})_{2}$

$85, \mathrm{R}=n-\mathrm{C}_{5} \mathrm{H}_{11}, \mathrm{R}^{\prime}=\mathrm{CH}_{2} \mathrm{CH}(n-\mathrm{Bu})_{2}$

86, $\mathrm{R}=n-\mathrm{C}_{5} \mathrm{H}_{11}, \mathrm{R}^{\prime}=\mathrm{CH}_{2} \mathrm{CH}(n-\mathrm{Pent})_{2}$

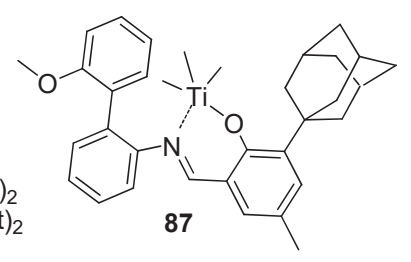

(c)<smiles>C=C(Br)CC(Br)C(C)C(Br)CC(Br)C=CC</smiles><smiles>C=C(Br)C(Br)CC(C)Br</smiles><smiles>CC#CCCC(Br)CC(C)Br</smiles>

(d)<smiles>[R]C(=C)CC([R])CC([R])C(C)C</smiles>

Scheme 7. (a) Metallacyclic mechanism of the coordination trimerization of $\alpha$-olefins; (b) Precatalysts of trimerization; (c) Product distribution for the trimerization of 1-hexene, catalyzed by 77/MAO [142]; (d) The main product of the trimerization of $\alpha$-olefins, catalyzed by 87 [146]. 
The metallacyclic mechanism was also proposed for the Ti (IV) complex 87 [146]. In the presence of $\mathrm{B}\left(\mathrm{C}_{6} \mathrm{~F}_{5}\right)_{3}$, this complex demonstrated more than $95 \%$ selectivity in the trimerization of 1-pentene, 1-hexene, and 1-decene with TONs $\sim 350\left(C_{5}, C_{6}\right)$ and $100\left(C_{10}\right)$. Among the trimers produced, ca. $85 \%$ were one regioisomer (Scheme $7 \mathrm{~d}$ ). The major olefin product is proposed to form by a tail-to-tail coupling, followed by 1,2-insertion and selective $\beta$-hydride elimination.

The theoretical aspects of three possible Cosse-Arlman, Green-Rooney, and metallacyclic mechanisms of $\alpha$-olefin oligomerization were studied theoretically by Copéret et al. [147]; the findings of this original article warrant further experimental studies.

Table 5. The regioisomers produced in the trimerization of 1-hexene with a range of catalysts [144].<smiles>C/C=C/C(Br)CC(C)Br</smiles>

A<smiles>C=C(Br)CC(Br)C(C)Br</smiles><smiles>BrC=CCC(Br)CCBr</smiles><smiles>[B]C(/C=C\C)CC(C)Br</smiles>

B<smiles>C=C(Br)CC(Br)CCBr</smiles><smiles>C=C(Br)C(Br)CC(C)Br</smiles><smiles>CC(Br)C(Br)C/C=C\Br</smiles><smiles>CC(Br)C(Br)C/C=C/Br</smiles><smiles>C=C(Br)C(Br)CCCBr</smiles>

$\mathrm{Bu}$<smiles>CC(C/C=C\Br)CCBr</smiles>
$\mathrm{K} \mathrm{Bu}$<smiles>[M]C(C=CCBr)CCCBr</smiles>

Regioisomer Abundance (\%)

\begin{tabular}{|c|c|c|c|c|c|c|c|c|c|c|c|c|}
\hline \multirow{2}{*}{ Cat. } & \multicolumn{12}{|c|}{ Regioisomer Abundance (\%) } \\
\hline & A & B & $\mathrm{C}$ & D & E & F & G & $\mathrm{H}$ & I & $\mathrm{K}$ & $\mathrm{L}$ & $\mathbf{M}$ \\
\hline 83 & 39.9 & 22.4 & 11.8 & 16.5 & 3.6 & 2.0 & 0.9 & 0.4 & 0.2 & 1.2 & 0.4 & 0.7 \\
\hline 84 & 38.5 & 19.2 & $15 . .5$ & 15.6 & 3.7 & 1.9 & 0.8 & 0.6 & 0.2 & 1.5 & 0.4 & 1.4 \\
\hline 85 & 46.9 & 15.3 & 3.0 & 19.7 & 8.3 & 3.1 & 1.5 & 0.3 & 0.2 & 0.7 & 0.3 & 0.7 \\
\hline 86 & 36.9 & 28.8 & 1.6 & 19.8 & 9.1 & 2.2 & 0.7 & 0.1 & $<0.1$ & 0.4 & $<0.1$ & 0.3 \\
\hline
\end{tabular}

Note that the Group 8 metal complexes demonstrated high efficiency in the oligomerization of ethylene [148-150] and norbornene [149-151]. The promising results in the selective oligomerization of propylene [152] and oligomerization/polymerization of 1-butene [153,154] and higher $\alpha$-olefins $[153,155-158]$ allow the consideration of such complexes as prospective post-metallocene oligomerization catalysts if the problem of moderate productivity could be solved.

\section{The Use of Methylenealkanes}

As shown above (see Section 2.1), methylenealkanes can be easily obtained by the zirconocene-catalyzed dimerization of $\alpha$-olefins. The yields of linear $\alpha$-olefin dimers typically exceed values of $90 \%$ in the event that an efficient pre-catalyst (i.e., 6 , Scheme $2 \mathrm{~b}$ ) is used [40]. In addition, methylenealkanes are unavoidable by-products of $\alpha$-olefin oligomerization (see Section 3 ). The problem of the utilization of $\alpha$-olefin dimers is of great relevance and importance $[33,41,159]$.

Methylenealkanes are close structural analogs of isobutylene. The presence of the reactive $C=C$ bond and a substantial difference in the environments of these unsaturated carbon atoms allows the consideration of methylenealkanes as prospective starting compounds for the regioselective synthesis of amphiphilic organic molecules and polymers. A number of possible directions for the synthetic use of methylenealkanes were demonstrated by us with the example of 5-methyleneundecane [41] (Figure 3). Below, we will refer to some of the notable examples of the use of methylenealkanes in the synthesis of organic compounds and polymers. 


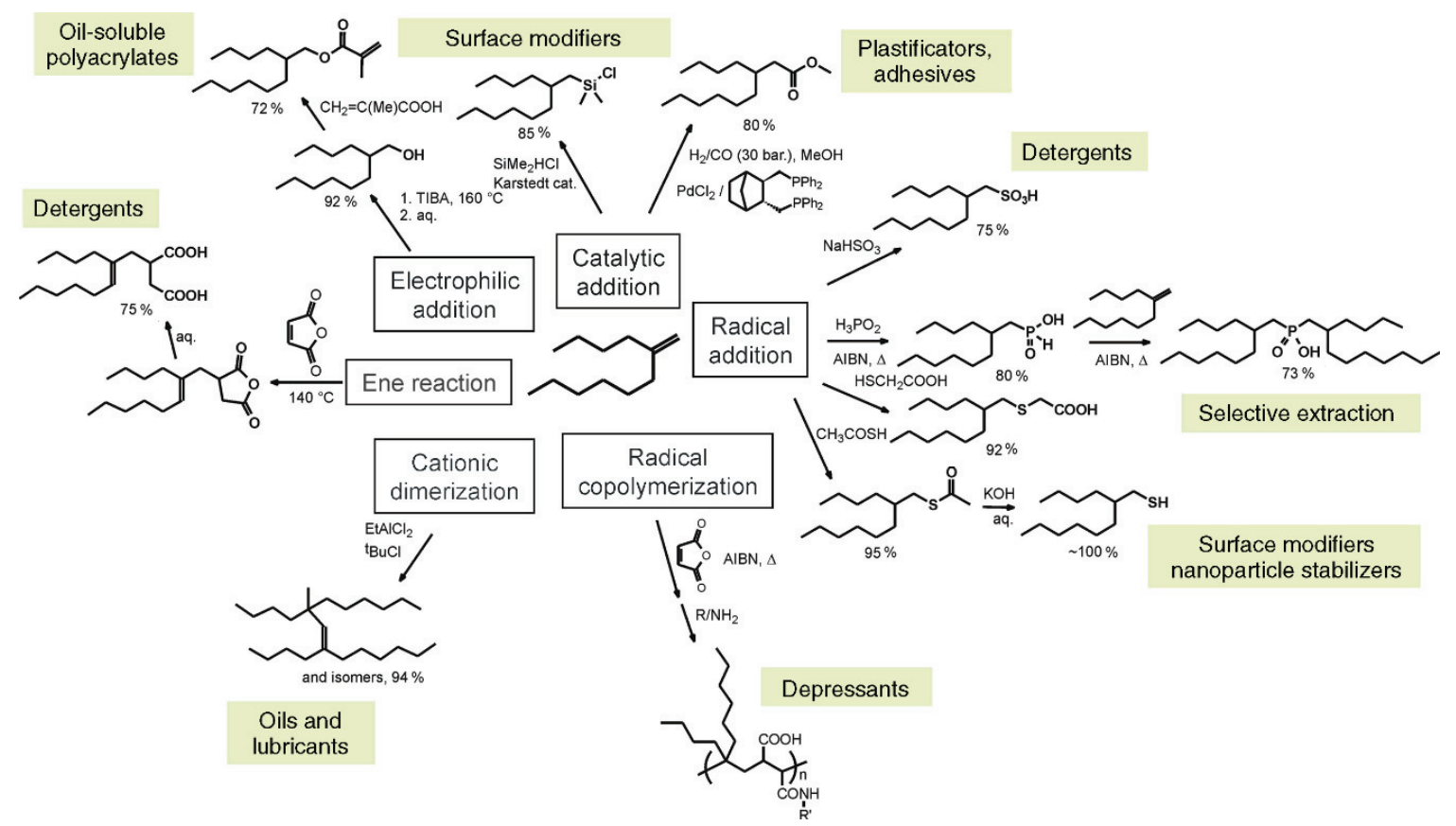

Figure 3. 5-methyleneundecane derivatives and their prospective applications. Reprinted with permission from [41]. Copyright (2017) De Gruyter.

\subsection{Free Radical Addition to Methylenealkanes}

The ease of free radical addition of $\mathrm{MeC}(\mathrm{O}) \mathrm{SH}$ using the method developed by Klotz et al. [160] was demonstrated on the example of 5-methyleneundecane (Figure 3) [41]. The closely related free-radical hydrophosphinylation of methylenealkanes was studied by Nifant'ev et al. in order to obtain hydrolytically stable extractants of rare-earth metals [161] (Scheme 8). Branched alkylphosphinic acids, obtained by the hydrophosphinylation of methylenealkanes, also demonstrated promising anti-wear properties [162].

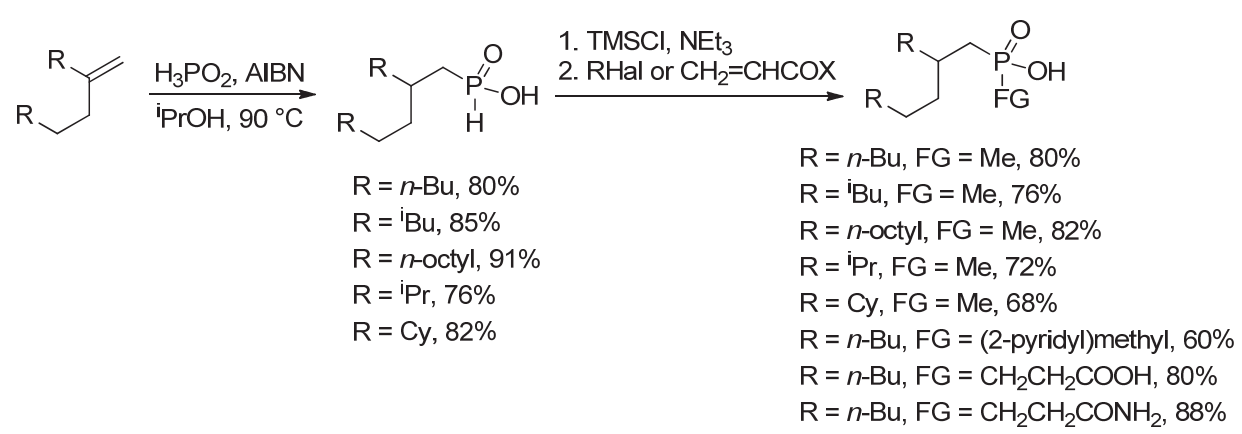

Scheme 8. Synthesis of alkylphosphinic and disubstituted phosphonic acids [161].

\subsection{Free Radical Polymerization of Methylenealkanes}

The ability of methylenealkanes to form copolymers with polar vinyl monomers was demonstrated by Yamago et al. with the example of the organotellurium-mediated living radical polymerization (TERP) of 6-methyleneundecane with acrylates; the tendency to form alternating copolymers was demonstrated [163]. Later, Nifant'ev et al. studied the copolymerization of a series of $\alpha$-olefin dimers with maleic anhydride [164]. In copolymerization experiments, performed in hydrocarbon media at $80-100^{\circ} \mathrm{C}$, azobisisobutyronitrile (AIBN)- or benzoyl peroxide (BPO)-initiated reactions resulted in the formation of copolymers with 1:1 comonomer ratios (Scheme 9), which would suggest an alternating nature of the reaction products. The post-modification of the copolymers obtained by the reactions 
with higher linear amines and alcohols (Scheme 9) resulted in copolymers with promising pour point depressant characteristics (Figure 4).

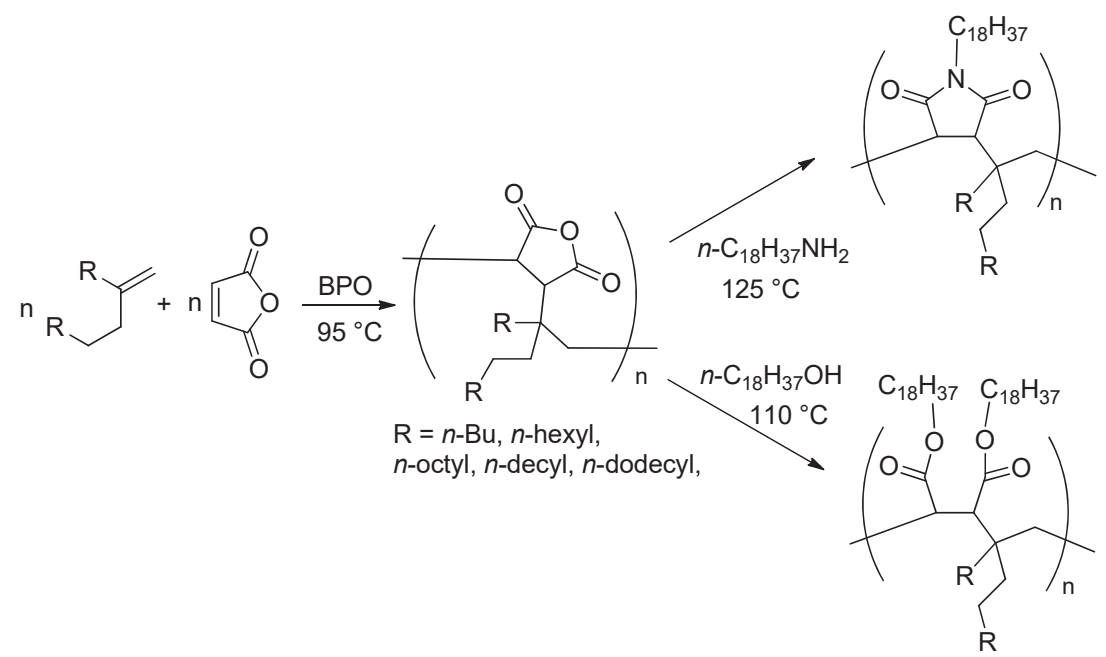

Scheme 9. Synthesis and modification of alternating copolymers of methylenealkanes and maleic anhydride [164].
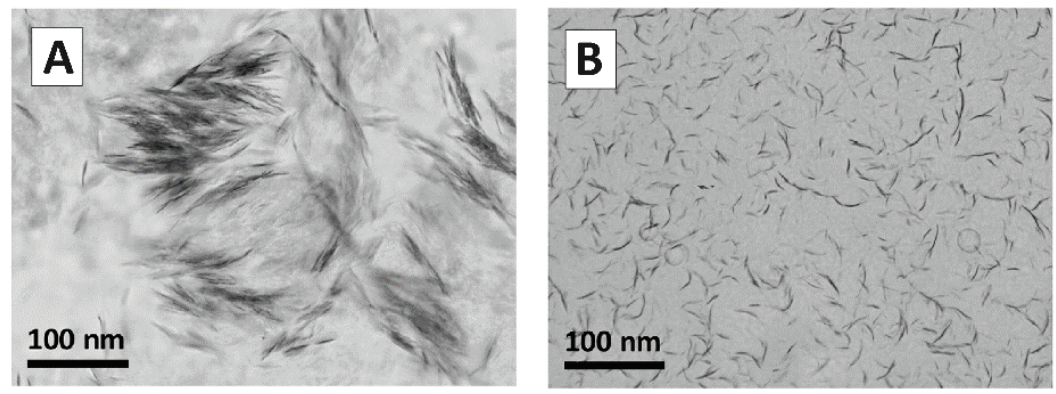

Figure 4. Morphology of paraffin crystals in the absence (A) and in the presence (B) of the pour point depressant, the octadecanol-modified copolymer of maleic anhydride and 9-methylenenonadecane [164].

The DFT modeling of copolymerization of olefins with maleic anhydride (MA) [165] confirmed the preference of the alternating reaction pathway for methylenealkanes. The possible Alder-ene side reaction (Figure 5a) was also studied; the results of calculations demonstrated that this thermally induced process cannot complete with alternating polymerization under the reaction conditions due to the relatively high level of the free energy of the corresponding transition state (Figure $5 b$ ). At elevated temperatures $\left(180-200{ }^{\circ} \mathrm{C}\right)$, this reaction proceeded within $4-6 \mathrm{~h}$ with good yields $[165,166]$; the Alder-ene adducts were used as a starting compounds in the synthesis of bis-succinimide friction modifiers for transmission fluids [166]. 


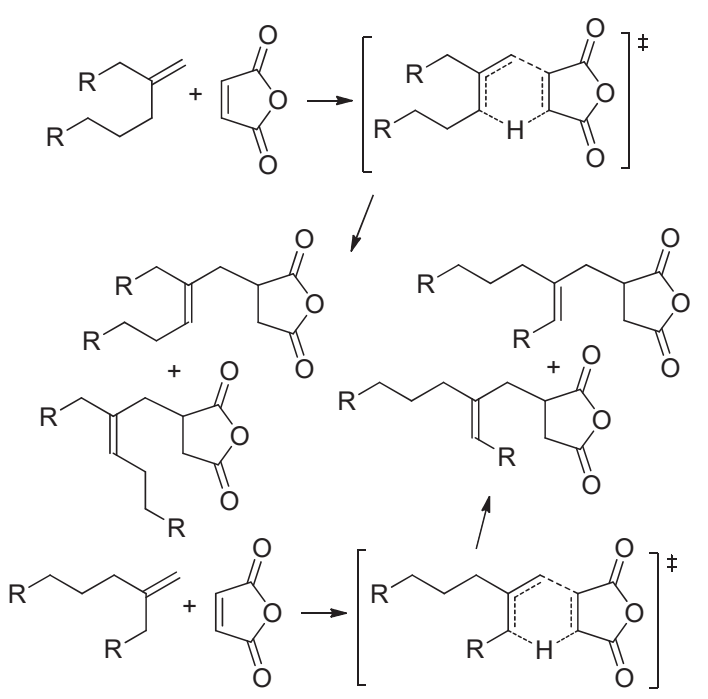

(a)

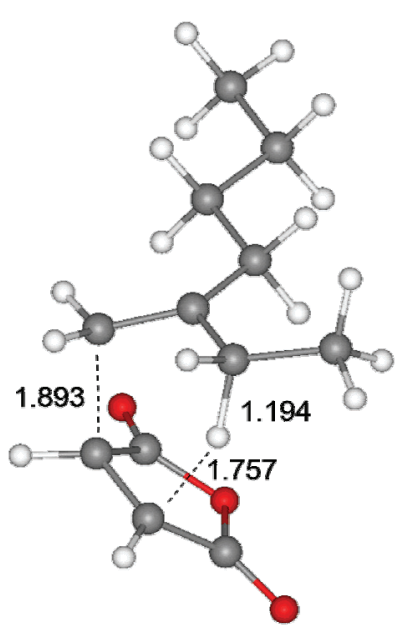

(b)

Figure 5. (a) The Alder-ene reaction between maleic anhydride (MA) and methylenealkanes; (b) Optimized geometry of the transition state of the Alder-ene reaction between MA and 3-methyleneheptane [165].

\subsection{Epoxydation and Related Reactions}

The reaction of methylenealkanes with $\mathrm{H}_{2} \mathrm{O}_{2} / \mathrm{HCOOH}$ resulted in the formation of the corresponding 1,2-diols with good yields (Scheme 10a) [167]. The acid-catalyzed rearrangement of the diols to the corresponding aldehydes, followed by the reaction with 1,2-diol, yielded branched acetals (Scheme 10a) [167]. Using toluene as a solvent, the reaction was stopped at the stage of the oxirane $[168,169]$; the acid-catalyzed rearrangement of the latter in the presence of $\mathrm{H}_{5} \mathrm{Mo}_{12} \mathrm{O}_{41} \mathrm{P}$ yielded the corresponding aldehyde (Scheme 10b) [168]. The first reaction sequence was the subject of the study of Harvey et al. [170] that represents a perfect example of neglect of the patent sources.

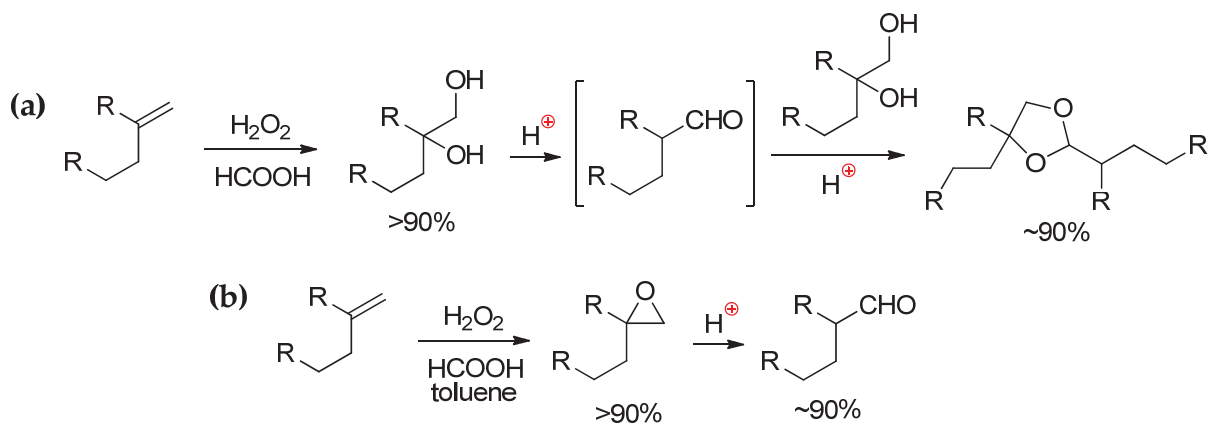

Scheme 10. Epoxidation of methylenealkanes with a formation of (a) 1,2-diols or (b) oxiranes; and related reactions.

\subsection{Methylenealkanes as Alkylating Reagents}

In contrast with the acid-catalyzed oligomerization of linear $\alpha$-olefins, accompanied by rearrangements with the formation of a large number of isomeric products, methylenealkanes in the presence of acids form products with the same carbon skeleton (Scheme 11). Kissin and Schwab achieved $90 \%$ conversions of 5 -methyleneundecane after $5 \mathrm{~h}$ of the reaction at $60{ }^{\circ} \mathrm{C}$ with $87 \%$ "dimer of dimer" selectivity using silica-supported $\mathrm{EtAlCl}_{2}$ [47]. The $\mathrm{C}_{40}$ fraction obtained by the dimerization of 9-methylenenonadecane (dimer of 1-decene) had promising viscosity characteristics (KV ${ }^{100}$ of 6.4 CSt and VI of 147) [47]. The product of the hydrogenolysis of this compound represents a promising low-viscosity PAO base [171]. Nifant'ev et al. proposed a more efficient catalytic system (1 mol \% 
of tert- $\mathrm{BuCl}$ and $\mathrm{EtAlCl}_{2}$ ) that allowed the achievement of $98 \%$ conversions of 1-hexene, 1-octene, and 1-decene dimers after $2 \mathrm{~h}$ of the reaction at $-30{ }^{\circ} \mathrm{C}$ [91]. Methylenealkanes were also used in the electrophilic alkylation of diphenylamine, catalyzed by acid-treated clays or ionic liquids [172].

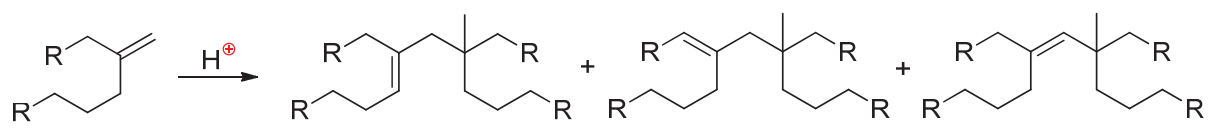

Scheme 11. Electrophilic dimerization of $\alpha$-olefin dimers, methylenealkanes $[47,91,171]$.

\subsection{Catalytic Transformations of Methylenealkanes}

The hydroformylation of 9-methylenenonadecane in the presence of $0.01 \mathrm{~mol} \%$ of $\left(\mathrm{Ph}_{3} \mathrm{P}\right)_{3} \mathrm{Rh}(\mathrm{CO}) \mathrm{H}$ resulted in the formation of the corresponding aldehyde (Scheme 12a) with a 76\% yield [173]. A phosphine-modified cobalt catalyst was less efficient (66\% yield) [174]. The best results, a $92 \%$ yield with $99 \%$ selectivity, were obtained using a (acac) $\mathrm{Rh}(\mathrm{CO})_{2} / \mathrm{PPh}_{3}$ catalyst [175]. The closely related methoxycarbonylation of methylenealkanes (Scheme 12b) was accompanied by the isomerization of the starting hydrocarbons; the rational design of the Pd/diphosphine catalyst had made it possible to reach average yields of the branched methyl esters [176]. The esters obtained were used in the synthesis of Group 5 oil base stocks with promising viscosity characteristics [177].

Catalytic hydrosilylation was another potentially significant process that allowed the obtaining of highly branched disiloxane with promising viscosity characteristics [178] (Scheme 12c). The same products can be clearly obtained by the hydrolysis of the branched chlorosilanes easily accessible via the hydrosilylation by $\mathrm{Me}_{2} \mathrm{SiHCl}$, catalyzed by a Karstedt catalyst [41] (see Figure 3).

(a)

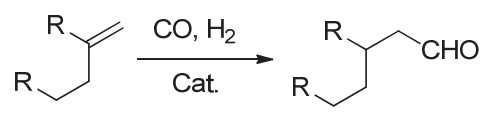

(b)

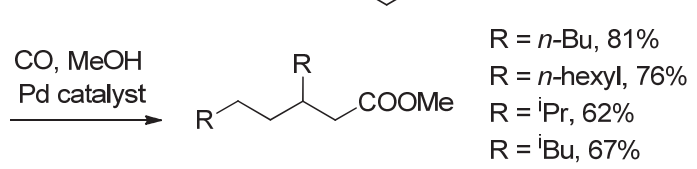

(c)

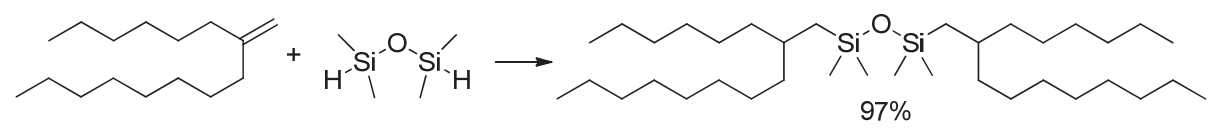

Scheme 12. Catalytic transformations of methylenealkanes: (a) Hydrofirmylation [173,175]; (b) Methoxycarbonylation [176]; (c) Hydrosilylation [178].

\section{Oils and Lubricants Based on Coordination Oligomers of $\alpha$-Olefins}

As was demonstrated previously, the viscosity properties such as VI and pour point $(P P)$ of poly- $\alpha$-olefin (PAO) oil base stocks depend strongly on the architecture of the constituent hydrocarbons $[16,17,179,180]$. Linear hydrocarbons, petroleum waxes, (Figure 6, A), which are present in large amounts in Group I and II oils, have high PPs. Consequently, these oils have limitations for their use since they cannot provide secure low-temperature engine start and transmission performance. Group III oil base hydrocarbons, which contain compounds with short branches (Figure 6, B) and cycloalkanes (Figure 6, C), also have relatively high $P P$ s and low-temperature viscosity values. Long-chain branched hydrocarbons (Figure 6, D) are characterized by low PPs and high Vis and therefore represent the most prospective group of hydrocarbons for use as high-grade bases of engine oils and transmission fluids. 


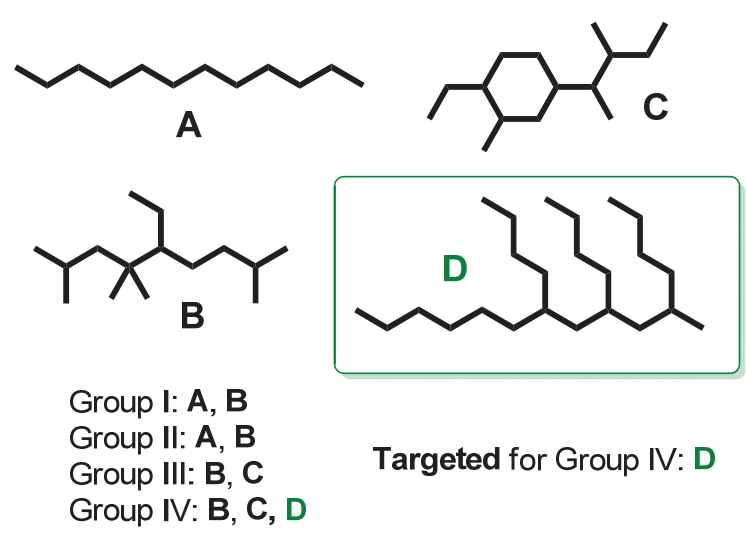

Figure 6. Main structural types of hydrocarbons-components of oils. Reprinted with permission from [91]. Copyright (2018) Elsevier B.V.

As mentioned in the Introduction section, cationic oligomerization is accompanied by a huge number of rearrangements, including peculiar reactions proposed by Shubkin [14] and studied later by Gee et al. [15] (Scheme 13). The products of metallocene-catalyzed oligomerization are vinylidene-type $\alpha$-olefin oligomers with uniform molecular structures (Scheme 1a). A gas chromatogram of the $C_{20}$ fraction of the products of the cationic oligomerization of 1-decene (Figure 7a) [15] confirms the complexity of the process with the formation of large number of reaction products; the difference between this grim picture and the gas chromatogram of the products of zirconocene-catalyzed oligomerization (Figure 7b) [103] clearly establishes the prospects of "metallocene" oligomers in terms of the structural homogeneity.

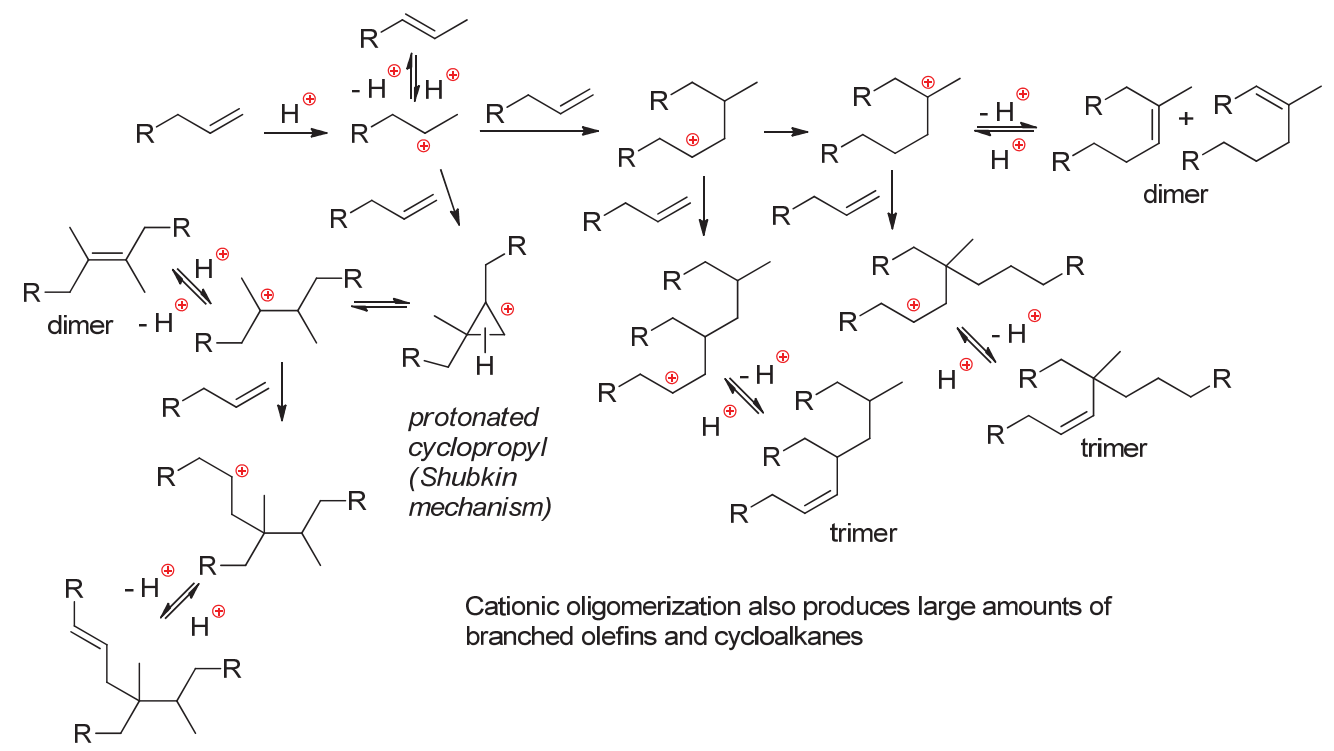

Scheme 13. Rearrangements in the cationic oligomerization of $\alpha$-olefins [14,15]. 
(a)

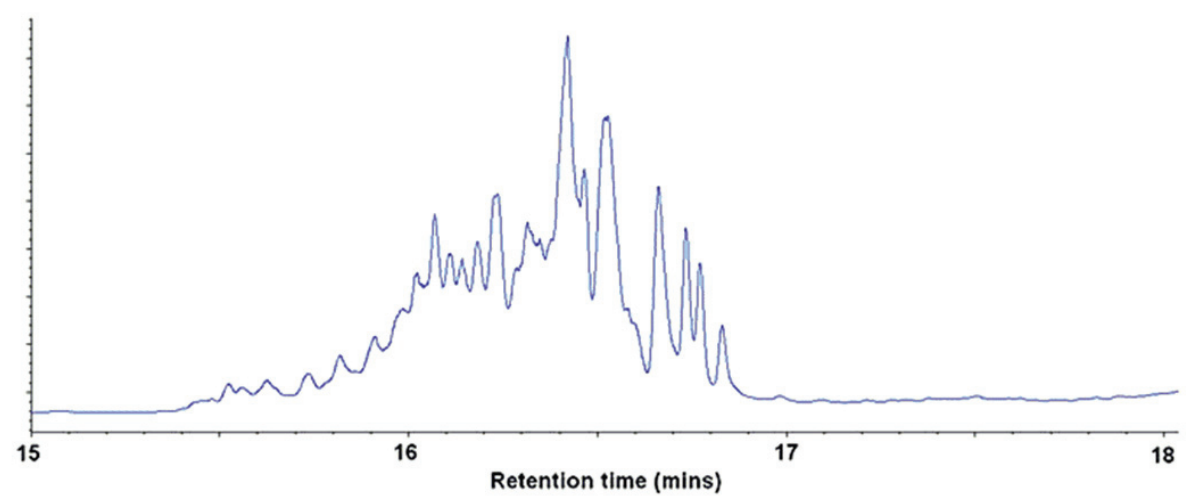

(b)
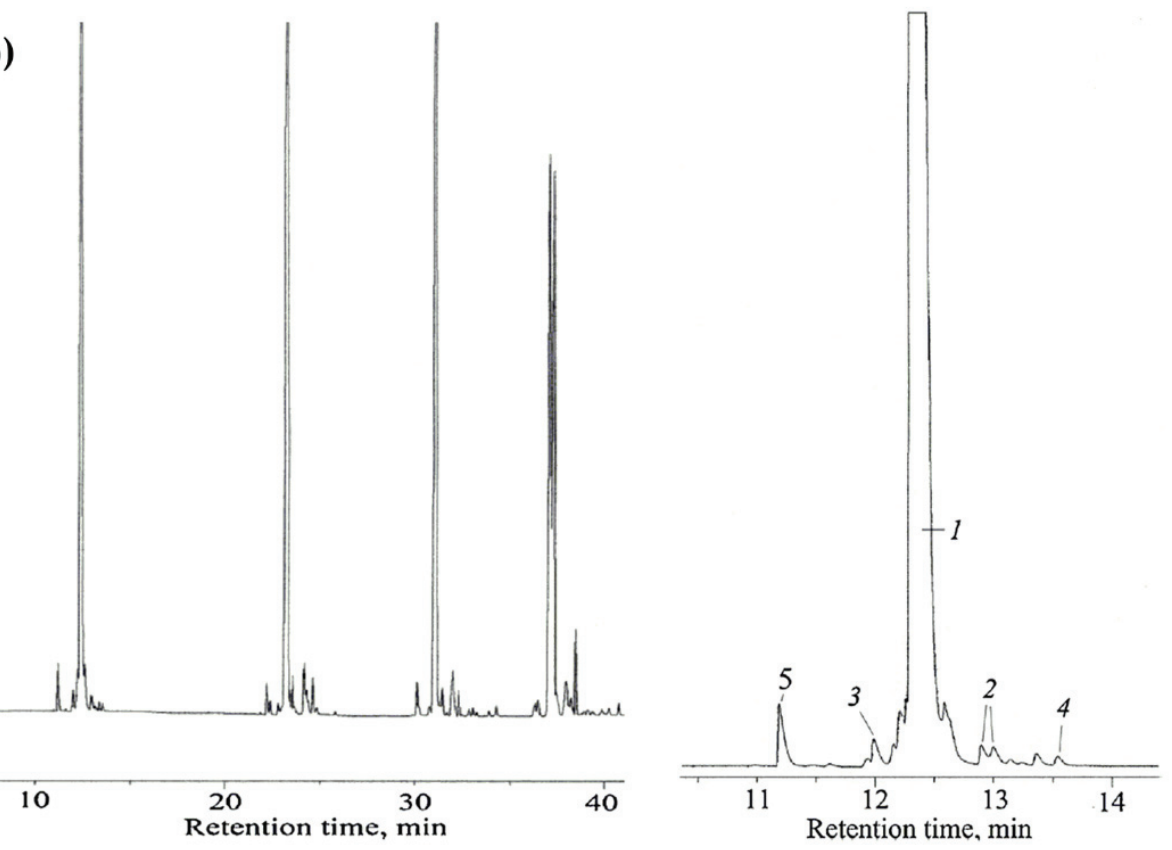

Figure 7. (a) Gas chromatogram showing peaks for $\mathrm{C}_{20}$ olefin isomers during acid-catalyzed 1-decene oligomerization. Reprinted with permission from [15]. Copyright (2012) Wiley-VCH Verlag GmbH \& Co; (b) Gas chromatorgams $\left(\mathrm{C}_{12}-\mathrm{C}_{30}\right.$ range, left) and dimer range (right) of 1-hexene oligomers produced with the $8 / \mathrm{MAO}$ catalyst at $70{ }^{\circ} \mathrm{C}$. Reprinted with permission from [103]. Copyright (2019) Elsevier B. V.

The transformation of the $\alpha$-olefin oligomers to PAO base stocks necessitates catalytic hydrogenation. Electrophilic oligomerization led to the partial formation of tetra-substituted olefins (Scheme 13); the presence of $>\mathrm{C}=\mathrm{C}<$ fragments significantly hampers the complete hydrogenolysis of double bonds. The ease of the hydrogenation is an additional benefit of the coordination $\alpha$-olefin oligomers. The viscosity characteristics of the $\alpha$-olefin oligomers obtained using electrophilic and metallocene catalysts and hygrogenated oligomers (PAO basestocks) are given in Table 6 and illustrated by Figure 8 [91]. These data clearly demonstrate that 1-hexene oligomers have little or no value for use as PAO base stocks due to low viscosity indices and high pour point values. The oligomers of 1-octene prepared by the zirconocene-catalyzed reaction have medium viscosity and outperform the 1-octene oligomers obtained in the presence of $\mathrm{BF}_{3}-\mathrm{ROH}$ according to viscosity indices, while possessing the same low-temperature kinematic viscosity. The oligomers of 1-decene synthesized by the zirconocene-catalyzed process significantly outperform the electrophilic oligomers obtained in the presence of $\mathrm{BF}_{3}-\mathrm{ROH}$ and can be considered as base stocks for modern PAOs.

The above pertains to oligomers obtained by the zirconocene-catalyzed process (Scheme 1a); however, as mentioned in Section 3.5, coordination oligomerization can proceed by a metallacyclic 
mechanism with the formation of products with different molecular structures (Scheme 8). The comparison of the viscosity characteristics of 1-decene trimers obtained by the zirconocene-catalyzed reaction and a metallacyclic process ( $\mathrm{Cr}$ catalysts $\mathbf{7 9}, \mathbf{8 0}, \mathbf{8 2})$ suggests that metallocene catalysis is preferable for the production of PAO oil base stocks.

Table 6. Viscosity characteristics of $\alpha$-olefin oligomers. ${ }^{1}$

\begin{tabular}{|c|c|c|c|c|c|c|c|}
\hline No. of C Atoms & Oligomer & $\begin{array}{c}K V^{-40} \\
\mathrm{sSt}\end{array}$ & $\begin{array}{c}K V^{40} \\
\text { sSt }\end{array}$ & $\begin{array}{c}K V^{100} \\
\mathrm{sSt}\end{array}$ & $V I$ & $P P,{ }^{\circ} \mathrm{C}$ & Ref. \\
\hline 12 & $\mathrm{H} 2 \mathrm{H}$ & 9.00 & 1.28 & - & - & -73 & [91] \\
\hline 16 & $\mathrm{O} 2$ & - & 2.6 & - & - & & [91] \\
\hline 16 & $\mathrm{O} 2 \mathrm{H}$ & 53.1 & 2.82 & - & - & -43 & [91] \\
\hline 18 & H3 & & 3.12 & & & & [91] \\
\hline 18 & H3h & 167.2 & 3.57 & - & - & -94 & [91] \\
\hline 18 & H3h $\left(\mathrm{BF}_{3}\right.$ catalyst $)$ & 165 & 3.8 & 1.4 & - & $<-55$ & [16] \\
\hline 20 & D2 & & 4.55 & 1.7 & 14 & & [91] \\
\hline 20 & D2h & & 5.30 & - & 14 & -7 & [91] \\
\hline 24 & H4 & & 7.55 & 2.10 & 62 & & [91] \\
\hline 24 & H4h & 1335 & 8.93 & 2.28 & 46 & -79 & [91] \\
\hline 24 & H4h $\left(\mathrm{BF}_{3}\right.$ catalyst $)$ & 1780 & 9.8 & 2.6 & 94 & & [16] \\
\hline 24 & $\mathrm{H} 22 \mathrm{~h}$ & 3030 & 12.4 & 2.72 & 27 & -71 & [91] \\
\hline 24 & O3 & & 6.5 & 2.06 & 114 & & [91] \\
\hline 24 & O3h & 552.1 & 7.56 & 2.20 & 92 & -86 & [91] \\
\hline 24 & $\mathrm{O} 3 \mathrm{H}\left(\mathrm{BF}_{3}\right.$ catalyst $)$ & 580 & 8.0 & 2.3 & 92 & $<-55$ & [16] \\
\hline 30 & H5 & & 17.55 & 3.6 & 76 & & [91] \\
\hline 30 & H5h & 6798 & 19.2 & 3.8 & 76 & -67 & [91] \\
\hline 30 & $\mathbf{H} 5 \mathbf{h}\left(\mathrm{BF}_{3}\right.$ catalyst $)$ & 7850 & 18.1 & 3.8 & 96 & & [16] \\
\hline 30 & D3 & & 14.61 & 3.65 & 140 & & [91] \\
\hline 30 & D3h (catalyst 79) & & 12.2 & 3.2 & 126 & & [143] \\
\hline 30 & D3h (catalyst 80) & & 13.0 & 3.3 & 131 & & [143] \\
\hline 30 & D3h (catalyst 82) & & 12.1 & 3.2 & 137 & & [143] \\
\hline 30 & D3h & 1897 & 15.05 & 3.70 & 137 & -75 & [91] \\
\hline 30 & D3h [EBTHI]Zr - borate & & 13.5 & 3.39 & 128 & & [52] \\
\hline 30 & D3h $\left(\mathrm{BF}_{3}\right.$ catalyst $)$ & 2070 & 15.6 & 3.7 & 122 & $<-55$ & [16] \\
\hline 32 & $\mathrm{O} 4$ & & 13.94 & 3.44 & 125 & & [91] \\
\hline 32 & O4h & 3135 & 18.4 & 4.0 & 115 & -74 & [91] \\
\hline 32 & $\mathrm{O} 4 \mathrm{~h}\left(\mathrm{BF}_{3}\right.$ catalyst $)$ & 4750 & 20.0 & 4.1 & 106 & $<-55$ & [16] \\
\hline 32 & $\mathrm{O} 22$ & & 28.1 & 5.4 & 130 & & [91] \\
\hline 32 & $\mathrm{O} 22 \mathrm{~h}$ & 6374 & 29.4 & 5.4 & 119 & -68 & [91] \\
\hline 40 & O5 & & 32.9 & 6.05 & 132 & & [91] \\
\hline 40 & O5h & 11651 & 36.2 & 6.4 & 129 & -68 & [91] \\
\hline 40 & O5h $\left(\mathrm{BF}_{3}\right.$ catalyst $)$ & 10225 & 30.9 & 5.6 & 124 & & [16] \\
\hline 40 & D4 & & 31.3 & 6.03 & 142 & & [91] \\
\hline 40 & D4h & 8631 & 34.5 & 6.52 & 145 & -66 & [91] \\
\hline 30 & D4h $\left(\mathrm{BF}_{3}\right.$ catalyst $)$ & 7475 & 29.0 & 5.7 & 141 & & [16] \\
\hline 40 & D22 & & 29.43 & 5.90 & 150 & & [91] \\
\hline 40 & D22h & 15615 & 42.0 & 7.25 & 136 & -52 & [91] \\
\hline
\end{tabular}

\footnotetext{
${ }^{1}$ Abbreviations used: $\mathbf{H}-1$-hexene, $\mathrm{O}-1$-octene, $\mathrm{D}-1$-decene oligomers; 2-5-DP $\mathrm{n}$ value; 22—dimer of dimer;
} h-hydrogenated. 


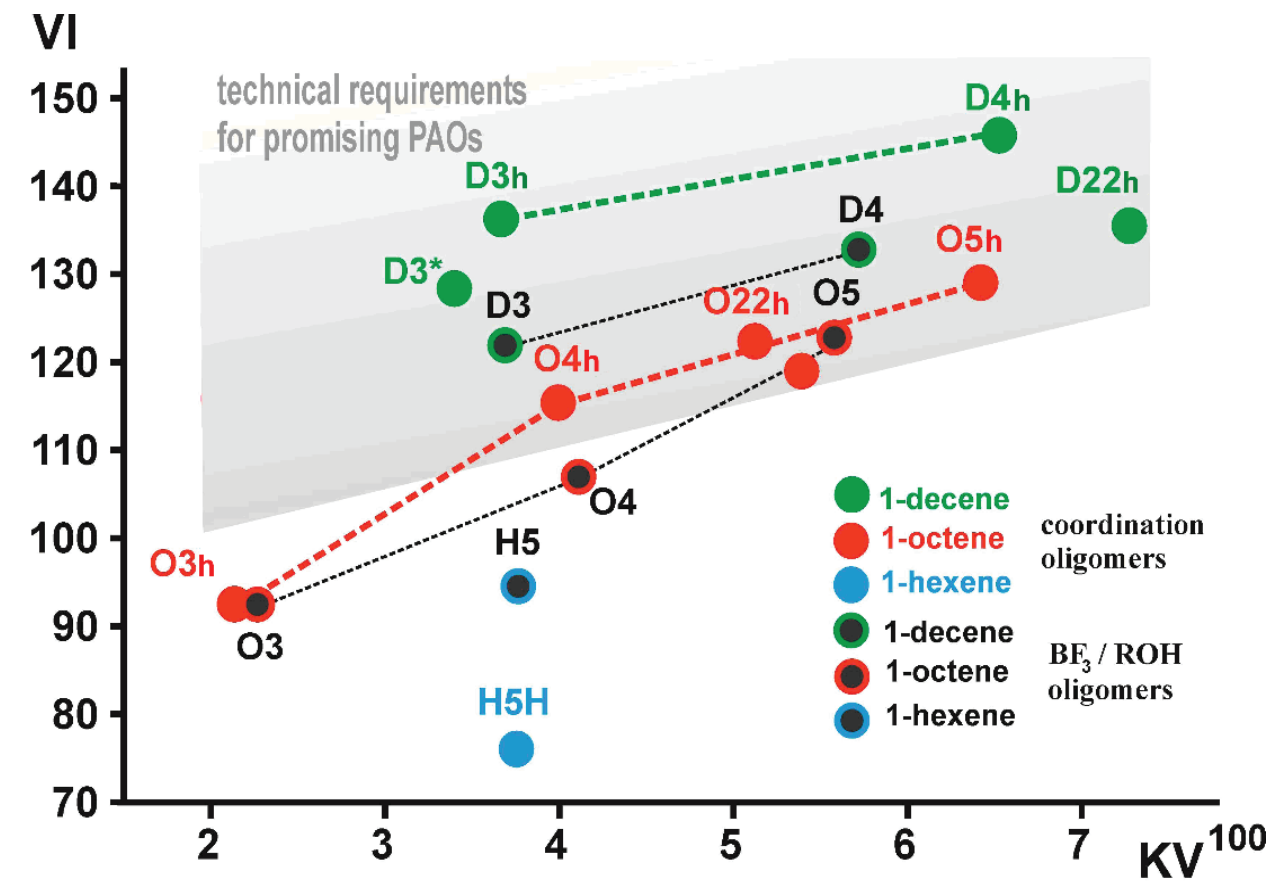

Figure 8. Viscosity characteristics of 1-decene (green), 1-octene (red), and 1-hexene (blue) oligomers obtained by coordination polymerization [91] and in the presence of $\mathrm{BF}_{3}-\mathrm{ROH}$ [16]. D3*: decene trimer obtained using [EBTHI] $\mathrm{ZrCl}_{2}-\mathrm{TIBA}$-perfluoroborate [52]. The range of technical requirements for promising poly- $\alpha$-olefin oils (PAOs) was limited to the segment corresponding to $\mathrm{VI}=100$ for $\mathrm{KV}^{100}=$ 2 and VI $=120$ for $\mathrm{KV}^{100}=6$. Reprinted with permission from [91]. Copyright (2018) Elsevier B. V.

\section{Conclusions}

Thus, it can be concluded that the zirconocene-catalyzed oligomerization of higher $\alpha$-olefins represents a flexible and resource-efficient method for the synthesis of methylenealkanes (vinylidene dimers of $\alpha$-olefins, $>90 \%$ yields with $>98 \%$ selectivities) and oligomers with a given $D P_{\mathrm{n}}$. A broader view of the importance of the molecular structure and molecular design that emerged over recent years resulted in the development of advanced "metallocene" technologies for poly- $\alpha$-olefin oils and lubricants by leading petrochemical companies such as Exxon, Idemitsu, and Mobil. The further progress in this field is related to the creation of the novel metallocene catalysts in order to achieve enhanced thermal stability, catalytic productivity, and selectivity in the synthesis of the desired oligomer fractions. Our recent research on 1-octene oligomerization and polymerization [80] further strongly suggests the high potential of "heterocenes" as a new generation of single-site catalysts of $\alpha$-olefin oligomerization. We have obtained early results that are extremely promising.

The problem of the utilization of methylenealkanes, which are imminent side products of metallocene-catalyzed oligomerization, is still relevant. The fundamental difference between methylenealkanes and isobutylene consists of the ability of methylenealkanes to isomerize with the formation of more stable branched olefins with $>\mathrm{C}=\mathrm{CH}-$ fragments. The research and development of the catalysts and processes without such isomerization is an actual affront to researchers' professionalism.

Author Contributions: Conceptualization, P.I.; methodology, I.N. and P.I.; writing-original draft preparation, P.I.; writing-review and editing, I.N. and P.I.; visualization, P.I.; supervision, I.N.; project administration, I.N.; funding acquisition, I.N. All authors have read and agreed to the published version of the manuscript.

Funding: This work was carried out within the State Program of TIPS RAS.

Conflicts of Interest: The authors declare no conflict of interest. 


\section{References}

1. Qiao, J.; Guo, M.; Wang, L.; Liu, D.; Zhang, X.; Yu, L.; Song, W.; Liu, Y. Recent advances in polyolefin technology. Polym. Chem. 2011, 2, 1611-1623. [CrossRef]

2. Shamiri, A.; Chakrabarti, M.H.; Jahan, S.; Hussain, M.A.; Kaminsky, W.; Aravind, P.V.; Yehye, W.A. The Influence of Ziegler-Natta and Metallocene Catalysts on Polyolefin Structure, Properties, and Processing Ability. Materials 2014, 7, 5069-5108. [CrossRef] [PubMed]

3. Collins, R.A.; Russell, A.F.; Mountford, P. Group 4 metal complexes for homogeneous olefin polymerisation: A short tutorial review. Appl. Petrochem. Res. 2015, 5, 153-171. [CrossRef]

4. Busico, V. Metal-catalysed olefin polymerisation into the new millennium: A perspective outlook. Dalton Trans. 2009, 41, 8794-8802. [CrossRef]

5. Sauter, D.W.; Taoufik, M.; Boisson, C. Polyolefins, a Success Story. Polymers 2017, 9, 185. [CrossRef]

6. Benda, R.; Bullen, J.; Plomer, A. Synthetics basics: Polyalphaolefins-Base fluids for high-performance lubricants. J. Synth. Lubric. 1996, 13, 41-57. [CrossRef]

7. Nicholas, C.P. Applications of light olefin oligomerization to the production of fuels and chemicals. Appl. Catal. A Gen. 2017, 543, 82-97. [CrossRef]

8. Ivchenko, P.V.; Nifant'ev, I.E.; Tavtorkin, A.V. Polyolefin Drag Reducing Agents (Review). Petrol. Chem. 2016, 56, 775-787. [CrossRef]

9. Nifant'ev, I.E.; Shlyakhtin, A.V.; Tavtorkin, A.N.; Korchagina, S.A.; Chinova, M.S.; Vinogradov, A.A.; Vinogradov, A.A.; Roznyatovsky, V.A.; Khaidapova, D.D.; Ivchenko, P.V. The synthesis of ultra-high molecular weight poly(1-hexene)s by low-temperature Ziegler-Natta precipitation polymerization in fluorous reaction media. Polymer 2018, 139, 98-106. [CrossRef]

10. Grumel, V.; Brull, R.; Pasch, H.; Raubenheimer, H.G.; Sanderson, R.; Wahner, U.M. Poly(pent-1-ene) synthesized with the syndiospecific catalyst $i-\operatorname{Pr}(\mathrm{Cp})(9-\mathrm{Flu}) \mathrm{ZrCl}_{2} / \mathrm{MAO}$. Macromol. Mater. Eng. 2002, 287, 559-564. [CrossRef]

11. Scupinska, J. Oligomerization of a-Olefins to Higher Oligomers. Chem. Rev. 1991, 91, 613-648. [CrossRef]

12. Ray, S.; Rao, P.V.C.; Choudary, N.V. Poly- $\alpha$-olefin-based synthetic lubricants: A short review on various synthetic routes. Lubric. Sci. 2012, 24, 23-44. [CrossRef]

13. Busca, G. Acid Catalysts in Industrial Hydrocarbon Chemistry. Chem. Rev. 2007, 107, 5366-5410. [CrossRef] [PubMed]

14. Shubkin, R.L.; Baylerian, M.S.; Maler, A.R. Olefin Oligomer Synthetic Lubricants: Structure and Mechanism of Formation. Ind. Eng. Chem. Prod. Res. Dev. 1980, 19, 15-19. [CrossRef]

15. Gee, J.C.; Small, B.L.; Hope, K.D. Behavior of protonated cyclopropyl intermediates during polyalphaolefin synthesis: Mechanism and predicted product distribution. J. Phys. Org. Chem. 2012, 25, 1409-1417. [CrossRef]

16. Brennan, J.A. Wide-temperature range synthetic hydrocarbon fluids. Ind. Eng. Chem. Prod. Res. Dev. 1980, 19, 2-6. [CrossRef]

17. Onopchenko, A.; Cupples, B.L.; Kresge, A.N. Boron fluoride-catalyzed oligomerization of alkenes: Structures, mechanisms, and properties. Ind. Eng. Chem. Prod. Res. Dev. 1983, 22, 182-191. [CrossRef]

18. Sun, H.; Shen, B.; Wu, D.; Guo, X.; Li, D. Supported Al-Ti bimetallic catalysts for 1-decene oligomerization: Activity, stability and deactivation mechanism. J. Catal. 2016, 339, 84-92. [CrossRef]

19. Hogg, J.M.; Coleman, F.; Ferrer-Ugalde, A.; Atkins, M.P.; Swadźba-Kwaśny, M. Liquid coordination complexes: A new class of Lewis acids as safer alternatives to $\mathrm{BF}_{3}$ in synthesis of polyalphaolefins. Green Chem. 2015, 17, 1831-1841. [CrossRef]

20. Antunes, B.M.; Rodrigues, A.E.; Lin, Z.; Portugal, I.; Silva, C.M. Alkenes oligomerization with resin catalysts. Fuel Proc. Technol. 2015, 138, 86-99. [CrossRef]

21. Wang, W.; Jiang, S.; Shen, Y.; Xia, S.; Xu, J. Synthesis of high viscosity index base stock and study on the lubricating properties. Ind. Lubricat. Tribol. 2016, 68, 52-56. [CrossRef]

22. Díaz-Rey, M.R.; Paris, C.; Martínez-Franco, R.; Moliner, M.; Martínez, C.; Corma, A. Efficient Oligomerization of Pentene into Liquid Fuels on Nanocrystalline Beta Zeolites. ACS Catal. 2017, 7, 6170-6178. [CrossRef]

23. Nicholas, C.P.; Laipert, L.; Prabhakar, S. Oligomerization of Light Olefins to Gasoline: An Advanced NMR Characterization of Liquid Products. Ind. Eng. Chem. Res. 2016, 55, 9140-9146. [CrossRef] 
24. Echaroj, S.; Santikunaporn, M.; Chavadej, S. Transformation of Bioderived 1-Decanol to Diesel-like Fuel and Biobased Oil via Dehydration and Oligomerization Reactions. Energy Fuels 2017, 31, 9465-9476. [CrossRef]

25. Silva, M.S.A.F.; Fernandes, A.; Neves, P.; Antunes, M.M.; Rocha, S.M.; Ribeiro, M.F.; Silva, C.M.; Valente, A.A. Mesostructured Catalysts Based on the BEA Topology for Olefin Oligomerisation. ChemCatChem 2018, 10, 2741-2754. [CrossRef]

26. Kwon, M.-H.; Chae, H.-J.; Park, M.B. Oligomerization of 1-hexene over designed SBA-15 acid catalysts. J. Ind. Eng. Chem. 2018, 65, 397-405. [CrossRef]

27. Fehér, C.; Tomasek, S.; Hancsók, J.; Skoda-Földes, R. Oligomerization of light olefins in the presence of a supported Brønsted acidic ionic liquid catalyst. Appl. Catal. B Environ. 2018, 239, 52-60. [CrossRef]

28. Liu, P.; Redekop, E.; Gao, X.; Liu, W.-C.; Olsbye, U.; Somorjai, G.A. Oligomerization of Light Olefins Catalyzed by Brønsted-Acidic Metal-Organic Framework-808. J. Am. Chem. Soc. 2019, 141, 11557-11564. [CrossRef]

29. Silva, A.F.; Fernandes, A.; Antunes, M.M.; Ribeiro, M.F.; Silva, C.M.; Valente, A.A. Olefin oligomerisation over nanocrystalline MFI-based micro/mesoporous zeotypes synthesised via bottom-up approaches. Renew. Energy 2019, 138, 820-832. [CrossRef]

30. Zhang, L.; Ke, M.; Song, Z.; Liu, Y.; Shan, W.; Wang, Q.; Xia, C.; Li, C.; He, C. Improvement of the Catalytic Efficiency of Butene Oligomerization Using Alkali Metal Hydroxide-Modified Hierarchical ZSM-5 Catalysts. Catalysts 2018, 8, 298. [CrossRef]

31. Moon, S.; Chae, H.-J.; Park, M.B. Oligomerization of light olefins over ZSM-5 and beta zeolite catalysts by modifying textural properties. Appl. Catal. A Gen. 2018, 553, 15-23. [CrossRef]

32. Lee, D.; Kim, H.; Park, Y.-K.; Jeon, J.-K. Oligomerization of Butene Mixture over NiO/Mesoporous Aluminosilicate Catalyst. Catalysts 2018, 8, 456. [CrossRef]

33. Janiak, C.; Blank, F. Metallocene Catalysts for Olefin Oligomerization. Macromol. Symp. 2006, 236 , 14-22. [CrossRef]

34. Janiak, C. Metallocene and related catalysts for olefin, alkyne and silane dimerization and oligomerization. Coord. Chem. Rev. 2006, 250, 66-94. [CrossRef]

35. Belov, G.P. Selective Dimerization, Oligomerization, Homopolymerization and Copolymerization of Olefins with Complex Organometallic Catalysts. Russ. J. Appl. Chem. 2008, 81, 1655-1666. [CrossRef]

36. Slaugh, L.H.; Schoenthal, G.W. Vinylidene Olefin Process. U.S. Patent 4658078, 14 April 1987.

37. Christoffers, J.; Bergman, R.G. Catalytic Dimerization Reactions of $\alpha$-Olefins and $\alpha, \omega$-Dienes with $\mathrm{Cp}_{2} \mathrm{ZrCl}_{2} /$ Poly(methylalumoxane): Formation of Dimers, Carbocycles, and Oligomers. J. Am. Chem. Soc. 1996, 118, 4715-4716. [CrossRef]

38. Christoffers, J.; Bergman, R.G. Zirconocene-alumoxane (1:1)—A catalyst for the selective dimerization of $\alpha$-olefins. Inorg. Chim. Acta 1998, 270, 20-27. [CrossRef]

39. Thiele, S.; Erker, G. Adjusting the Features of Active Metallocene Ziegler Systems for Their Potential Use as Carbon-Carbon Coupling Catalysts in Organic Synthesis. Chem. Ber. 1997, 130, 201-207. [CrossRef]

40. Nifant'ev, I.E.; Vinogradov, A.A.; Vinogradov, A.A.; Ivchenko, P.V. Zirconocene-catalyzed dimerization of 1-hexene: Two-stage activation and structure-catalytic performance relationship. Catal. Commun. 2016, 79, 6-10. [CrossRef]

41. Nifant'ev, I.; Ivchenko, P.; Tavtorkin, A.; Vinogradov, A.; Vinogradov, A. Non-traditional Ziegler-Natta catalysis in $\alpha$-olefin transformations: Reaction mechanisms and product design. Pure Appl. Chem. 2017, 89, 1017-1032. [CrossRef]

42. Nifant'ev, I.E.; Vinogradov, A.A.; Vinogradov, A.A.; Churakov, A.V.; Ivchenko, P.V. Synthesis of zirconium(III) complex by reduction of $\mathrm{O}\left[\mathrm{SiMe}_{2}\left(\eta-\mathrm{C}_{5} \mathrm{H}_{4}\right)\right] \mathrm{ZrCl}_{2}$ and its selectivity in catalytic dimerization of hex-1-ene. Mendeleev Commun. 2018, 28, 467-469. [CrossRef]

43. Nifant'ev, I.E.; Vinogradov, A.A.; Vinogradov, A.A.; Ivchenko, P.V. The Method of the Syntheiss of Vinylidene Olefins. RU Patent 2652118, 25 April 2018.

44. Janiak, C.; Lange, K.C.H.; Marquardt, P. $\alpha$-Olefin oligomers with narrow molar mass distributions from zirconocene/methylaluminoxane catalysts-An examination of the structure-reactivity relationship. Macromol. Rapid Commun. 1995, 16, 643-650. [CrossRef]

45. Janiak, C.; Lange, K.C.H.; Marquardt, P.; Krüger, R.-P.; Hanselmann, R. Analyses of Propene and 1-Hexene Oligomers from Zirconocene/MAO Catalysts-Mechanistic Implications by NMR, SEC, and MALDI-TOF MS. Macromol. Chem. Phys. 2002, 203, 129-138. [CrossRef] 
46. Janiak, C.; Lange, K.C.H.; Marquardt, P. Alkyl-substituted cyclopentadienyl- and phospholyl-zirconium/MAO catalysts for propene and 1-hexene oligomerization. J. Mol. Catal. A Chem. 2002, 180, 43-58. [CrossRef]

47. Kissin, Y.V.; Schwab, F.C. Post-Oligomerization of alpha-Olefin Oligomers: A Route to Single-Component and Multicomponent Synthetic Lubricating Oils. J. Appl. Polym. Sci. 2009, 111, 273-280. [CrossRef]

48. Boccia, A.C.; Costabile, C.; Pragliola, S.; Longo, P. Selective Dimerization of $\gamma$-Branched $\alpha$-Olefins in the Presence of $C_{2}$ Group-4 Metallocene-Based Catalysts. Macromol. Chem. Phys. 2004, 205, 1320-1326. [CrossRef]

49. Bagheri, V.; Eisenberg, D.C.; Ratliff, K.S.; Benda, R.; Lanier, C.V. Oligomer oils and their manufacture. U.S. Patent 6548724, 15 April 2003.

50. Song, W.; Heilman, W.J. Synthesis of Poly-Alpha Olefin and Use Thereof. U.S. Patent Application 2003055184, 20 March 2003.

51. Fujikawa, S.; Yokota, K.; Okano, M.; Tsuji, M. Method for Producing $\alpha$-Olefin Oligomers and Lubricating Oil Compositions. U.S. Patent Application 2011207977, 25 August 2011.

52. Martin, R.W.; Deckman, D.E.; Kelly, K.J.; Emett, C.J.; Hagemeister, M.P.; Harrington, B.A.; Lin, C.-Y.; Matsunaga, P.T.; Ruff, C.J.; Stavens, K.B. Low Viscosity Engine Oil Compositions. U.S. Patent 9234150, 12 January 2016.

53. Emett, C.J.; Hagemeister, M.P.; Harrington, B.A.; Lin, C.Y.; Matsunaga, P.T.; Ruff, C.J.; Stavens, K.B. Process to Produce Improved Poly Alpha Olefin Composition. U.S. Patent 9365788, 14 June 2016.

54. van der Heijden, H.; Hessen, B.; Orpen, A.G. A Zwitterionic Zirconocene Alkyl Complex as a Single-Component $\alpha$-Olefin Dimerization Catalyst. J. Am. Chem. Soc. 1998, 120, 1112-1113. [CrossRef]

55. Takeuchi, K.; Fujikawa, S. Base Oil for Oil Drilling Fluid and Oil Drilling Fluid Composition. U.S. Patent Application 2011251445, 13 October 2011.

56. Fujikawa, S.; Okamoto, T.; Yokota, K. Process for Producing Unsaturated Hydrocarbon Compound. U.S. Patent 8119850, 21 February 2012.

57. Marks, T.J.; Yang, X. Homogeneous Alpha-Olefin Dimerization Catalysts. U.S. Patent 5500398, 19 March 1996.

58. Baldwin, S.M.; Bercaw, J.E.; Brintzinger, H.H. Alkylaluminum-Complexed Zirconocene Hydrides: Identification of Hydride-Bridged Species by NMR Spectroscopy. J. Am. Chem. Soc. 2008, 130, 17423-17433. [CrossRef]

59. Parfenova, L.V.; Kovyazin, P.V.; Tyumkina, T.V.; Islamov, D.N.; Lyapina, A.R.; Karchevsky, S.G.; Ivchenko, P.V. Reactions of bimetallic Zr, Al-hydride complexes with methylaluminoxane: NMR and DFT study. J. Organomet. Chem. 2017, 851, 30-39. [CrossRef]

60. Pankratyev, E.Y.; Tyumkina, T.V.; Parfenova, L.V.; Khalilov, L.M.; Khursan, S.L.; Dzhemilev, U.M. DFT Study on Mechanism of Olefin Hydroalumination by $\mathrm{XAlBu}_{2}{ }_{2}$ in the Presence of $\mathrm{Cp}_{2} \mathrm{ZrCl}_{2}$ Catalyst. I. Simulation of Intermediate Formation in Reaction of $\mathrm{HAlBu}_{2}{ }_{2}$ with $\mathrm{Cp}_{2} \mathrm{ZrCl}_{2}$. Organometallics 2009, 28, 968-977. [CrossRef]

61. Pankratyev, E.Y.; Tyumkina, T.V.; Parfenova, L.V.; Khalilov, L.M.; Khursan, S.L.; Dzhemilev, U.M. DFT and $\mathrm{Ab}$ Initio Study on Mechanism of Olefin Hydroalumination by XAlBu ${ }_{2}{ }_{2}$ in the Presence of $\mathrm{Cp}_{2} \mathrm{ZrCl}_{2}$ Catalyst. II. Olefin Interaction with Catalytically Active Centers. Organometallics 2011, 30, 6078-6089. [CrossRef]

62. Nifant'ev, I.; Vinogradov, A.; Vinogradov, A.; Karchevsky, S.; Ivchenko, P. Zirconocene-Catalyzed Dimerization of $\alpha$-Olefins: DFT Modeling of the Zr-Al Binuclear Reaction Mechanism. Molecules 2019, 24, 3565. [CrossRef] [PubMed]

63. Hanton, M.J.; Daubney, L.; Lebl, T.; Polas, S.; Smith, D.M.; Willemse, A. Selective dimerisation of $\alpha$-olefins using tungsten-based initiators. Dalton Trans. 2010, 7025-7037. [CrossRef]

64. Gunasekara, T.; Preston, A.Z.; Zeng, M.; Abu-Omar, M.M. Highly Regioselective $\alpha$-Olefin Dimerization Using Zirconium and Hafnium Amine Bis(phenolate) Complexes. Organometallics 2017, 36, 2934-2939. [CrossRef]

65. Leitch, D.C.; Lam, Y.C.; Labinger, J.A.; Bercaw, J.E. Upgrading Light Hydrocarbons via Tandem Catalysis: A Dual Homogeneous Ta/Ir System for Alkane/Alkene Coupling. J. Am. Chem. Soc. 2013, 135, 10302-10305. [CrossRef]

66. Leitch, D.C.; Labinger, J.A.; Bercaw, J.E. Scope and Mechanism of Homogeneous Tantalum/Iridium Tandem Catalytic Alkane/Alkene Upgrading using Sacrificial Hydrogen Acceptors. Organometallics 2014, 33, 3353-3365. [CrossRef]

67. Broene, R.D.; Brookhart, M.; Lamanna, W.M.; Volpe, A.F. Cobalt-Catalyzed Dimerization of $\alpha$-Olefins to Give Linear $\alpha$-Olefin Products. J. Am. Chem. Soc. 2005, 127, 17194-17195. [CrossRef] 
68. Kretschmer, W.P.; Troyanov, S.I.; Meetsma, A.; Hessen, B.; Teuben, J.H. Regioselective Homo- and Codimerization of $\alpha$-Olefins Catalyzed by Bis(2,4,7-trimethylindenyl)yttrium Hydride. Organometallics 1998, 17, 284-286. [CrossRef]

69. Piers, W.E.; Shapiro, P.J.; Bunel, E.E.; Bercaw, J.E. Coping with extreme Lewis acidity: Strategies for the synthesis of stable, mononuclear organometallic derivatives of scandium. Synlett 1990, 74-84. [CrossRef]

70. Huang, Q.; Chen, L.; Ma, L.; Fu, Z.; Yang, W. Synthesis and characterization of oligomer from 1-decene catalyzed by supported Ziegler-Natta catalysts. Eur. Polym. J. 2005, 41, 2909-2915. [CrossRef]

71. Huang, Q.; Chen, L.; Sheng, Y.; Ma, L.; Fu, Z.; Yang, W. Synthesis and characterization of oligomer from 1-decene catalyzed by $\mathrm{AlCl}_{3} / \mathrm{TiCl}_{4} / \mathrm{SiO}_{2} / \mathrm{Et}_{2} \mathrm{AlCl}$. J. Appl. Polym. Sci. 2006, 101, 584-590. [CrossRef]

72. Kawahara, N.; Saito, J.; Matsuo, S.; Kaneko, H.; Matsugi, T.; Toda, Y.; Kashiwa, N. Study on unsaturated structures of polyhexene, poly(4-methylpentene) and poly(3-methylpentene) prepared with metallocene catalysts. Polymer 2007, 48, 425-428. [CrossRef]

73. Brant, P.; Jiang, P.; Lovell, J.; Crowther, D. Termination events in sterically hindered metallocene-catalyzed olefin oligomerizations: Vinyl chain ends in oligooctenes. Organometallics 2016, 35, 2836-2839. [CrossRef]

74. Shao, H.; Wang, R.; Li, H.; Guo, X.; Jiang, T. Synthesis of low-molecular-weight poly- $\alpha$-olefins using silicon-bridged zirconocene catalyst for lubricant basestock. Arab. J. Chem. 2020, 13, 2715-2721. [CrossRef]

75. Kim, I.; Zhou, J.-M.; Chung, H. Higher $\alpha$-olefin polymerizations catalyzed by rac- $\mathrm{Me}_{2} \mathrm{Si}\left(1-\mathrm{C}_{5} \mathrm{H}_{2}-2-\mathrm{CH}_{3}-4-{ }^{t} \mathrm{Bu}\right)_{2} \mathrm{Zr}\left(\mathrm{NMe}_{2}\right)_{2} / \mathrm{Al}\left({ }^{\mathrm{i}} \mathrm{Bu}\right)_{3} /\left[\mathrm{Ph}_{3} \mathrm{C}\right]\left[\mathrm{B}\left(\mathrm{C}_{6} \mathrm{~F}_{5}\right)_{4}\right]$. J. Polym. Sci. Part A Polym. Chem. 2000, 38, 1687-1697. [CrossRef]

76. Zhao, X.; Odian, G.; Rossi, A. Kinetics, polymer molecular weights, and microstructure in zirconocene-catalyzed 1-hexene polymerization. J. Polym. Sci. Part A Polym. Chem. 2000, 38, 3802-3811. [CrossRef]

77. Babu, G.N.; Newmark, R.A.; Chien, J.C.W. Microstructure of Poly(1-hexene) Produced by ansa-Zirconocenium Catalysis. Macromolecules 1994, 27, 3383-3388. [CrossRef]

78. Grumel, V.; Brüll, R.; Pasch, H.; Raubenheimer, H.G.; Sanderson, R.; Wahner, U.M. Homopolymerization of Higher 1-Olefins with Metallocene/MAO Catalysts. Macromol. Mater. Eng. 2001, 286, 480-487. [CrossRef]

79. Babushkin, D.E.; Panchenko, V.N.; Brintzinger, H.H. Zirconium Allyl Complexes as Participants in Zirconocene-Catalyzed alpha-Olefin Polymerizations. Angew. Chem. Int. Ed. 2014, 53, 9645-9649. [CrossRef]

80. Nifant'ev, I.E.; Vinogradov, A.A.; Vinogradov, A.A.; Churakov, A.V.; Bagrov, V.V.; Kashulin, I.A.; Roznyatovsky, V.A.; Grishin, Y.K.; Ivchenko, P.V. The catalytic behavior of heterocenes activated by TIBA and MMAO under a low Al/Zr ratios in 1-octene polymerization. Appl. Catal. A: General 2019, 571, 12-24. [CrossRef]

81. Bryliakov, K.P.; Semikolenova, N.V.; Yudaev, D.V.; Zakharov, V.A.; Brintzinger, H.H.; Ystenes, M.; Rytter, E.; Talsi, E.P. ${ }^{1} \mathrm{H}-,{ }^{13} \mathrm{C}-\mathrm{NMR}$ and ethylene polymerization studies of zirconocene/MAO catalysts: Effect of the ligand structure on the formation of active intermediates and polymerization kinetics. J. Organomet. Chem. 2003, 683, 92-102. [CrossRef]

82. Tritto, I.; Donetti, R.; Sacchi, M.C.; Locatelli, P.; Zannoni, G. Dimethylzirconocene-Methylaluminoxane Catalyst for Olefin Polymerization: NMR Study of Reaction Equilibria. Macromolecules 1997, 30, 1247-1252. [CrossRef]

83. Babushkin, D.E.; Semikolenova, N.V.; Zakharov, V.A.; Talsi, E.P. Mechanism of dimethylzirconocene activation with methylaluminoxane: NMR monitoring of intermediates at high Al/Zr ratios. Macromol. Chem. Phys. 2000, 201, 558-567. [CrossRef]

84. Ehm, C.; Cipullo, R.; Budzelaar, P.H.M.; Busico, V. Role of TMA in polymerization. Dalton Trans. 2016, 45, 6847-6855. [CrossRef] [PubMed]

85. Trefz, T.K.; Henderson, M.A.; Linnolahti, M.; Collins, S.; McIndoe, J.S. Mass Spectrometric Characterization of Methylaluminoxane-Activated Metallocene Complexes. Chem. Eur. J. 2015, 21, 2980-2991. [CrossRef] [PubMed]

86. Talarico, G.; Budzelaar, P.H.M. Variability of Chain Transfer to Monomer Step in Olefin Polymerization. Organometallics 2008, 27, 4098-4107. [CrossRef]

87. Lieber, S.; Brintzinger, H.-H. Propene Polymerization with Catalyst Mixtures Containing Different ansa-Zirconocenes: Chain Transfer to Alkylaluminum Cocatalysts and Formation of Stereoblock Polymers. Macromolecules 2000, 33, 9192-9199. [CrossRef] 
88. Babushkin, D.E.; Brintzinger, H.H. Reactive intermediates formed during olefin polymerization by methylalumoxane-activated ansa-zirconocene catalysts: Identification of a chain-carrying intermediate by NMR methods. J. Am. Chem. Soc. 2010, 132, 452-453. [CrossRef]

89. Adamu, S.; Atiqullah, M.; Malaibari, Z.O.; Al-Harthi, M.A.; Emwas, A.-H.M.; Ul-Hamid, A. Metallocene-catalyzed ethylene- $\alpha$-olefin isomeric copolymerization: A perspective from hydrodynamic boundary layer mass transfer and design of MAO anion. J. Taiwan Ins. Chem. Eng. 2016, 60, 92-105. [CrossRef]

90. Nifant'ev, I.E.; Vinogradov, A.A.; Vinogradov, A.A.; Bezzubov, S.I.; Ivchenko, P.V. Catalytic oligomerization of $\alpha$-olefins in the presence of two-stage activated zirconocene catalyst based on 6,6-dimethylfulvene 'dimer'. Mendeleev Commun. 2017, 27, 35-37. [CrossRef]

91. Nifant'ev, I.E.; Vinogradov, A.A.; Vinogradov, A.A.; Sedov, I.V.; Dorokhov, V.G.; Lyadov, A.S.; Ivchenko, P.V. Structurally uniform 1-hexene, 1-octene, and 1-decene oligomers: Zirconocene/MAO-catalyzed preparation, characterization, and prospects of their use as low-viscosity low-temperature oil base stocks. Appl. Catal. A Gen. 2018, 549, 40-50. [CrossRef]

92. Ivchenko, P.V.; Nifant'ev, I.E.; Vinogradov, A.A.; Krut'ko, D.P.; Shandryuk, G.A. Crosslinked $\alpha$-olefin-diene copolymers prepared using a metallocene catalyst deposited on the surface of $\mathrm{SiO}_{2}$-modified $\mathrm{Fe}_{3} \mathrm{O}_{4}$ : Ferromagnetic oil sponges. Polym. Sci. Ser. B 2017, 59, 83-90. [CrossRef]

93. DiMaio, A.J.; Baranski, J.R.; Budworth, J.G.; Gillis, D.J. Process for Producing Liquid Polyalphaolefin Polymer, Metallocene Catalyst therefor, the Resulting Polymer and Lubricant Containing Same. U.S. Patent 6858767, 22 February 2005.

94. Londaitsbehere, A.; Cuenca, T.; Mosquera, M.E.G.; Cano, J.; Milione, S.; Grassi, A. 1,3-Double Siloxo-Bridged Zirconium Metallocene for Propene and 1-Hexene Regioselective Oligomerization. Organometallics 2012, 31, 2108-2111. [CrossRef]

95. Welle, A.; Wassenaar, J.; Slawinski, M. Use of a Metallocene Catalyst to Produce a Polyalpha-Olefin. U.S. Patent 9688792, 27 June 2017.

96. Wu, M.M.-S.; Baugh, L.S.; Canich, J.A.; Chee, C.S.; Hagemeister, M.P.; Jackson, A.; Jiang, P.; Lee, G.H.; Lo, F.Y.-K.; Rucker, S.P.; et al. Polyalpha-Olefin Compositions and Processes to Produce the Same. U.S. Patent Application 2009005279, 01 January 2009.

97. Small, B.L.; Hope, K.D.; Masino, A.P.; McDaniel, M.P.; Buck, R.M.; Beaulieu, W.B.; Yang, Q.; Baralt, E.J.; Netemeyer, E.J.; Kreischer, B. Oligomerization of Alpha Olefins Using Metallocene-SSA Catalyst Systems and Use of the Resultant Polyalphaolefins to Prepare Lubricant Blends. U.S. Patent 8536391, 17 September 2013.

98. Di Maio, A.J. Process for the Oligmerization of Alpha-Olefins Having Low Unsaturation. U.S. Patent 7129306, 31 October 2006.

99. Minami, H.; Egawa, T. Process for Producing a Polymer of an Alpha-Olefin and Lubricant. U.S. Patent Application 2002143113, 03 October 2002.

100. Iimure, Y.; Hirano, H.; Tohi, Y.; Upakawa, N. Synthetic Lubricating Oil and Lubricating Oil Composition. U.S. Patent 7795194, 14 September 2010.

101. Shao, H.; Li, H.; Lin, J.; Jiang, T.; Guo, X.; Li, J. Metallocene-catalyzed oligomerizations of 1-butene and $\alpha$-olefins: Toward synthetic lubricants. Eur. Polym. J. 2014, 59, 208-217. [CrossRef]

102. Kissin, Y.V. Detailed Kinetics of 1-Hexene Oligomerization Reaction with $(n-\mathrm{Bu}-\mathrm{Cp})_{2} \mathrm{ZrCl}_{2}-\mathrm{MAO}$ Catalyst. Macromol. Chem. Phys. 2009, 210, 1241-1246. [CrossRef]

103. Kissin, Y.V. Oligomerization reactions of 1-hexene with metallocene catalysts: Detailed data on reaction chemistry and kinetics. Mol. Catal. 2019, 463, 87-93. [CrossRef]

104. Dong, S.Q.; Mi, P.K.; Xu, S.; Zhang, J.; Zhao, R.D. Preparation and Characterization of Single-Component Poly- $\alpha$-olefin Oil Base Stocks. Energy Fuels 2019, 33, 9796-9804. [CrossRef]

105. De Rosa, C.; Auriemma, F.; Di Capua, A.; Resconi, L.; Guidotti, S.; Camurati, I.; Nifant'ev, I.E.; Laishevtsev, I.P. Structure-Property Correlations in Polypropylene from Metallocene Catalysts: Stereodefective, Regioregular Isotactic Polypropylene. J. Am. Chem. Soc. 2004, 126, 17040-17049. [CrossRef]

106. Nifant'ev, I.E.; Laishevtsev, I.P.; Ivchenko, P.V.; Kashulin, I.A.; Guidotti, S.; Piemontesi, F.; Camurati, I.; Resconi, L.; Klusener, P.A.A.; Rijsemus, J.J.H.; et al. C 1 -Symmetric Heterocyclic Zirconocenes as Catalysts for Propylene Polymerization, 1. ansa-Zirconocenes with Linked Dithienocyclopentadienyl-Substituted Cyclopentadienyl Ligands. Macromol. Chem. Phys. 2004, 205, 2275-2291. [CrossRef] 
107. Resconi, L.; Guidotti, S.; Camurati, I.; Frabetti, R.; Focante, F.; Nifant'ev, I.E.; Laishevtsev, I.P. C ${ }_{1}$-Symmetric Heterocyclic Zirconocenes as Catalysts for Propylene Polymerization, 2. ansa-Zirconocenes with Linked Dithienocyclopentadienyl-Substituted Indenyl Ligands. Macromol. Chem. Phys. 2005, 206, 1405-1438. [CrossRef]

108. Ryabov, A.N.; Gribkov, D.V.; Izmer, V.V.; Voskoboynikov, A.Z. Zirconium Complexes with Cyclopentadienyl Ligands Involving Fused a Thiophene Fragment. Organometallics 2002, 21, 2842-2855. [CrossRef]

109. Yang, X.; Stern, C.L.; Marks, T.J.; Cationic Metallocene Polymerization Catalysts. Synthesis and Properities of the Cationic Metallocene Polymerization Catalysts. Synthesis and Properities of the First Base-Free Zirconocene Hydride. Angew. Chem. Int. Ed. 1992, 31, 1375-1377. [CrossRef]

110. Chen, E.Y.-X.; Marks, T.J. Cocatalysts for Metal-Catalyzed Olefin Polymerization: Activators, Activation Processes, and Structure-Activity Relationships. Chem. Rev. 2000, 100, 1391-1434. [CrossRef] [PubMed]

111. Chen, M.-C.; Roberts, J.A.S.; Seyam, A.M.; Li, L.; Zuccaccia, C.; Stahl, N.G.; Marks, T.J. Diversity in Weakly Coordinating Anions. Mono- and Polynuclear Halo(perfluoroaryl)metalates as Cocatalysts for Stereospecific Olefin Polymerization: Synthesis, Structure, and Reactivity. Organometallics 2006, 25, 2833-2850. [CrossRef]

112. Park, J.H.; Jang, Y.E.; Jeon, J.Y.; Go, M.J.; Lee, J.; Kim, S.K.; Lee, S.-I.; Lee, B.Y. Preparation of ansa-metallocenes for production of poly ( $\alpha$-olefin) lubricants. Dalton Trans. 2014, 43, 10132-10138. [CrossRef]

113. Wu, M.M.; Coker, C.L.; Walzer, J.F.; Jiang, P. Process to Produce Low Viscosity Poly-Alfa-Olefins. U.S. Patent 8207390, 26 June 2012.

114. Wu, M.M.; Coker, C.L.; Walzer, J.F.; Jiang, P.; Rucker, S.P. Process to Produce High Viscosity Fluids. U.S. Patent 7989670, 02 August 2011.

115. Wu, M.M.; Pafford, B.J.; Stavens, K.B. Polyalphaolefins by Oligomerization and Isomerization. U.S. Patent Application 2014323665, 30 October 2014.

116. Hagemeister, M.P.; Jiang, P.; Wu, M.M.; Yang, N. Production of Shear-Stable High Viscosity PAO. U.S. Patent 9365663, 14 June 2016.

117. Wu, M.M.; Hagemeister, M.P.; Yang, N. Process to Produce Polyalphaolefins. U.S. Patent 8513478, 20 August 2013.

118. Jiang, H.; Yu, K. Catalytic polymerization of 1-decene using a silicon-bridged metallocene system. Petrol. Sci. Technol. 2017, 35, 1451-1456. [CrossRef]

119. Katayama, K.; Noda, H.; Shimizu, H.; Okano, M. Alpha-Olefin (co)Polymer, Hydrogenated $\alpha$-Olefin (co)Polymer and Lubricating Oil Composition Containing Them. U.S. Patent Application 2012302481, 29 November 2012.

120. Shimizu, H.; Katayama, K.; Noda, H.; Okano, M. 1-Octene, 1-Decene, 1-Dodecene Ternary Copolymer and Lubricants Therewith. U.S. Patent Application 2014256997, 11 September 2014.

121. Shimizu, H.; Katayama, K.; Noda, H.; Okano, M. 1-Octene/1-Decene Copolymer and Libricating-Oil Composition Containing Same. U.S. Patent Application 2014309151, 16 October 2014.

122. Patil, A.O.; Bodige, S.; Luo, S.; Chu, J.W.; Stavens, K.; Harrrington, B.A. Ultra High Viscosity Synthetic Base Stocks and Process for Preparing Same. U.S. Patent Application 2014213834, 31 July 2014.

123. Patil, A.O.; Bodige, S. Synthetic Libricant Basestocks and Methods of Preparation Thereof. U.S. Patent 9422497, 23 August 2016.

124. Wu, M.M.; Rucker, S.P.; Canich, A.M. Process for Producing Novel Synthetic Basestocks. U.S. Patent 9701595 , 11 July 2017.

125. Courtiade, M.; Sanson, J.; Welle, A.; Slawinski, M.; Wassenaar, J. Method for Preparing Low-Viscosity Lubricating Polyolefins. U.S. Patent Application 2017226441, 10 August 2017.

126. Gibson, V.C.; Spitzmesser, S.K. Advances in Non-Metallocene Olefin Polymerization Catalysis. Chem. Rev. 2003, 103, 283-315. [CrossRef]

127. Gibson, V.C.; Redshaw, C.; Solan, G.A. Bis(imino)pyridines: Surprisingly Reactive Ligands and a Gateway to New Families of Catalysts. Chem. Rev. 2007, 107, 1745-1776. [CrossRef]

128. Budagumpi, S.; Kim, K.-H.; Kim, I. Catalytic and coordination facets of single-site non-metallocene organometallic catalysts with $\mathrm{N}$-heterocyclic scaffolds employed in olefin polymerization. Coord. Chem. Rev. 2011, 255, 2785-2809. [CrossRef]

129. Sydora, O.L.; Jones, T.C.; Small, B.L.; Nett, A.J.; Fischer, A.A.; Carney, M.J. Selective Ethylene Tri-/Tetramerization Catalysts. ACS Catal. 2012, 2, 2452-2455. [CrossRef] 
130. Agapie, T. Selective ethylene oligomerization: Recent advances in chromium catalysis and mechanistic investigations. Coord. Chem. Rev. 2011, 255, 861-880. [CrossRef]

131. van Leeuwen, P.W.N.M.; Clément, N.D.; Tschan, M.J.-L. New processes for the selective production of 1-octene. Coord. Chem. Rev. 2011, 255, 1499-1517. [CrossRef]

132. Small, B.L. Discovery and Development of Pyridine-bis(imine) and Related Catalysts for Olefin Polymerization and Oligomerization. Acc. Chem. Res. 2015, 48, 2599-2611. [CrossRef] [PubMed]

133. Baier, M.C.; Zuideveld, M.A.; Mecking, S. Post-Metallocenes in the Industrial Production of Polyolefins. Angew. Chem. Int. Ed. 2014, 53, 9722-9744. [CrossRef]

134. Alferov, K.A.; Belov, G.P.; Meng, Y. Chromium catalysts for selective ethylene oligomerization to 1-hexene and 1-octene: Recent results. Appl. Catal. A Gen. 2017, 542, 71-124. [CrossRef]

135. Raucoules, R.; de Bruin, T.; Raybaud, P.; Adamo, C. Theoretical Unraveling of Selective 1-Butene Oligomerization Catalyzed by Iron-Bis(arylimino)pyridine. Organometallics 2009, 28, 5358-5367. [CrossRef]

136. Majoumo-Mbe, F.; Lönnecke, P.; Volkis, V.; Sharma, M.; Eisen, M.S.; Hey-Hawkins, E. Oligomerization of $\alpha$-olefins by the dimeric nickel bisamido complex $\left[\mathrm{Ni}\left\{1-\mathrm{N}\left(\mathrm{PMes}_{2}\right)-2-\mathrm{N}\left(\mu-\mathrm{PMes}_{2}\right) \mathrm{C}_{6} \mathrm{H}_{4}-\mathrm{K}^{3} \mathrm{~N}, \mathrm{~N}^{\prime}, \mathrm{P},-\mathrm{K}^{1} \mathrm{P}^{\prime}\right\}\right]_{2}$ activated by methylalumoxane (MAO). J. Organomet. Chem. 2008, 693, 2603-2609. [CrossRef]

137. Dagorne, S.; Bellemin-Laponnaz, S.; Romain, C. Neutral and Cationic N-Heterocyclic Carbene Zirconium and Hafnium Benzyl Complexes: Highly Regioselective Oligomerization of 1-Hexene with a Preference for Trimer Formation. Organometallics 2013, 32, 2736-2743. [CrossRef]

138. Despagnet-Ayoub, E.; Takase, M.K.; Henling, L.M.; Labinger, J.A.; Bercaw, J.E. Mechanistic Insights on the Controlled Switch from Oligomerization to Polymerization of 1-Hexene Catalyzed by an NHC-Zirconium Complex. Organometallics 2015, 34, 4707-4716. [CrossRef]

139. Nakata, N.; Nakamura, K.; Ishii, A. Highly efficient and 1,2-regioselective method for the oligomerization of 1-hexene promoted by zirconium precatalysts with [OSSO]-type bis(phenolate) ligands. Organometallics 2018, 37, 2640-2644. [CrossRef]

140. Nakata, N.; Nakamura, K.; Nagaoka, S.; Ishii, A. Carbazolyl-Substituted [OSSO]-Type Zirconium(IV) Complex as a Precatalyst for the Oligomerization and Polymerization of $\alpha$-Olefins. Catalysts 2019, 9, 528. [CrossRef]

141. Manyik, R.M.; Walker, W.E.; Wilson, T.P. A soluble chromium-based catalyst for ethylene trimerization and polymerization. J. Catal. 1977, 47, 197-209. [CrossRef]

142. Köhn, R.D.; Haufe, M.; Kociok- Köhn, G.; Grimm, S.; Wasserscheid, P.; Keim, W. Selective Trimerization of $\alpha$-Olefins with Triazacyclohexane Complexes of Chromium as Catalysts. Angew. Chem. Int. Ed. 2000, 39, 4337-4339. [CrossRef]

143. Wasserscheid, P.; Grimm, S.; Köhn, R.D.; Haufe, M. Synthesis of Synthetic Lubricants by Trimerization of 1-Decene and 1-Dodecene with Homogeneous Chromium Catalysts. Adv. Synth. Catal. 2001, 343, 814-818. [CrossRef]

144. Coxon, A.G.N.; Köhn, R.D. Efficient 1-Hexene Trimerization with Triazacyclohexane Chromium Catalysts and Detailed Product Analysis by ${ }^{13}$ C NMR. ACS Catal. 2016, 6, 3008-3016. [CrossRef]

145. Luo, S.; Patil, A.O. Processes for Preparing Low Viscosity Lubricating Oil Base Stocks. U.S. Patent 8889931, 18 November 2014.

146. Sattler, A.; Labinger, J.A.; Bercaw, J.E. Highly Selective Olefin Trimerization Catalysis by a Borane-Activated Titanium Trimethyl Complex. Organometallics 2013, 32, 6899-6902. [CrossRef]

147. Gordon, C.P.; Shirase, S.; Yamamoto, K.; Andersen, R.A.; Eisenstein, O.; Copéret, C. NMR chemical shift analysis decodes olefin oligo- and polymerization activity of $\mathrm{d}^{0}$ group 4 metal complexes. Proc. Natl. Acad. Sci. USA 2018, 115, E5867-E5876. [CrossRef]

148. 148 Speiser, F.; Braunstein, P.; Saussine, L. Catalytic Ethylene Dimerization and Oligomerization: Recent Developments with Nickel Complexes Containing P,N-Chelating Ligands. Acc. Chem. Res. 2005, 38, 784-793. [CrossRef]

149. 149 Yao, Z.-J.; Deng, W. Half-sandwich late transition metal complexes based on functionalized carborane ligands. Coord. Chem. Rev. 2016, 309, 21-35. [CrossRef]

150. 150 Yao, Z.-J.; Li, P.; Li, K.; Deng, W. Synthesis, structure and catalytic polymerization activity of half-sandwich cyclometallated iridium complexes. Appl. Organomet. Chem. 2018, 32, e4239. [CrossRef] 
151. 151 Antonov, A.A.; Semikolenova, N.V.; Zakharov, V.A.; Zhang, W.; Wang, Y.; Sun, W.-H.; Talsi, E.P.; Bryliakov, K.P. Vinyl Polymerization of Norbornene on Nickel Complexes with Bis(imino)pyridine Ligands Containing Electron-Withdrawing Groups. Organometallics 2012, 31, 1143-1149. [CrossRef]

152. 152 Mlinar, A.N.; Keitz, B.K.; Gygi, D.; Bloch, E.D.; Long, J.R.; Bell, A.T. Selective Propene Oligomerization with Nickel(II)-Based Metal-Organic Frameworks. ACS Catal. 2014, 4, 717-721. [CrossRef]

153. 153 Behr, A.; Bayrak, Z.; Peitz, S.; Stochniol, G.; Maschmeyer, D. Oligomerization of 1-butene with a homogeneous catalyst system based on allylic nickel complexes. RSC Adv. 2015, 5, 41372-41376. [CrossRef]

154. 154 Forget, S.; Olivier-Bourbigou, H.; Delcroix, D. Homogeneous and Heterogeneous Nickel-Catalyzed Olefin Oligomerization: Experimental Investigation for a Common Mechanistic Proposition and Catalyst Optimization. ChemCatChem 2017, 9, 2408-2417. [CrossRef]

155. 155 Guo, L.; Dai, S.; Sui, X.; Chen, C. Palladium and Nickel Catalyzed Chain Walking Olefin Polymerization and Copolymerization. ACS Catal. 2016, 6, 428-441. [CrossRef]

156. 156 Hu, H.; Gao, H.; Chen, D.; Li, G.; Tan, Y.; Liang, G.; Zhu, F.; Wu, Q. Ligand-Directed Regioselectivity in Amine-Imine Nickel-Catalyzed 1-Hexene Polymerization. ACS Catal. 2015, 5, 122-128. [CrossRef]

157. 157 O'Connor, K.S.; Lamb, J.R.; Vaidya, T.; Keresztes, I.; Klimovica, K.; LaPointe, A.M.; Daugulis, O.; Coates, G.W. Understanding the Insertion Pathways and Chain Walking Mechanisms of $\alpha$-Diimine Nickel Catalysts for $\alpha$-Olefin Polymerization: A ${ }^{13} \mathrm{C}$ NMR Spectroscopic Investigation. Macromolecules 2017, 50, 7010-7027. [CrossRef]

158. 158 Ojwach, S.O.; Darkwa, J. Perspective and future prospects of tandem olefin oligomerization and Friedel-Crafts alkylation reactions catalyzed by iron, cobalt, nickel and palladium complexes. Catal. Sci. Technol. 2019, 9, 2078-2096. [CrossRef]

159. Aruleswaran, N.R.; Sharko, P.T.; Crump, J.G. Vinylidene Dimer Derivatives. U.S. Patent Application 2015284350, 08 October 2015.

160. Kerbal, A. Synthesis and Glutathione S-Transferase Structure-Affinity Relationships of Nonpeptide and Peptidase-Stable Glutathione Analogues. J. Med. Chem. 1998, 41, 2278-2288. [CrossRef]

161. Nifant'ev, I.E.; Minyaev, M.E.; Tavtorkin, A.N.; Vinogradov, A.A.; Ivchenko, P.V. Branched alkylphosphinic and disubstituted phosphinic and phosphonic acids: Effective synthesis based on $\alpha$-olefin dimers and applications in lanthanide extraction and separation. RSC Adv. 2017, 7, 24122-24128. [CrossRef]

162. Nifant'ev, I.E.; Lyadov, A.S.; Tavtorkin, A.N.; Vinogradov, A.A.; Kochubeev, A.A.; Ivchenko, P.V. Branched alkylphosphinic acids demonstrate explicit anti-wear effect. Mendeleev Commun. 2019, 29, 558-560. [CrossRef]

163. Mishima, E.; Tamura, T.; Yamago, S. Controlled Copolymerization of Acrylate and 6-Methyleneundecane by Organotellurium-Mediated Living Radical Polymerization (TERP). Macromolecules 2012, 45, 2989-2994. [CrossRef]

164. Nifant'ev, I.E.; Vinogradov, A.A.; Bondarenko, G.N.; Korchagina, S.A.; Shlyakhtin, A.V.; Roznyatovsky, V.A.; Ivchenko, P.V. Copolymers of Maleic Anhydride and Methylene Alkanes: Synthesis, Modification, and Pour Point Depressant Properties. Polym. Sci. Ser. B 2018, 60, 469-480. [CrossRef]

165. Nifant'ev, I.; Vinogradov, A.; Vinogradov, A.; Ivchenko, P. DFT Modeling of the Alternating Radical Copolymerization and Alder-Ene Reaction between Maleic Anhydride and Olefins. Polymers 2020, 12, 744. [CrossRef]

166. Shanker, S.K.; Shiga, M.; Fuchi, M.; Nakagawa, T. Lubricating Oil Composition and Method for Operating Automatic Transmission. U.S. Patent Application 2013029890, 31 January 2013.

167. Sato, H.; Okamoto, T. Dioxolane Derivative and Method for Producing Same. EP Patent 1792900, 06 June 2007.

168. Sato, H.; Kashiwamura, T.; Ikeda, Y. Process for Producing Aldehyde with 2-Postiion Branched Long-Chain Alkyl. EP Patent 1908746, 09 April 2008.

169. Sato, H.; Shishikura, A. Alkyl Acetal Compound, Process for Producing the Same, and Lubricatiog Oil Composition. U.S. Patent Application 2008234152, 25 September 2008.

170. Harvey, B.G.; Meylemansa, H.A.; Quintana, R.L. Synthesis of renewable plasticizer alcohols by formal anti-Markovnikov hydration of terminal branched chain alkenes via a borane-free oxidation/reduction sequence. Green Chem. 2012, 14, 2450-2456. [CrossRef]

171. Sato, H.; Kamimura, H. Process for Producing Saturated Aliphatic Hydrocarbon Compound, and Lubricant Composition. U.S. Patent 8373011, 12 February 2013.

172. Patil, A.O. Diphenylamine Functionalization of Poly-Alpha-Olefins. U.S. Patent 7847030, 07 December 2009. 
173. Sato, H.; Kashiwamura, T.; Okamoto, T.; Yokota, K. Carbonyl Compound Containing Long-Chain Branched Alkyl Group. U.S. Patent 7402610, 22 July 2008.

174. De Kraker, A.R. Olefin Oligomer Composition. U.S. Patent 8383869, 26 February 2013.

175. Patil, A.; Lewis, K.G.; Bodige, S.; Zushma, S. Ester Compounds, Lubricating Oil Composition Containing Same and Process for Making Same. U.S. Patent Application 2019062663, 28 February 2019.

176. Nafant'ev, I.E.; Sevostyanova, N.T.; Batashev, S.A.; Vinogradov, A.A.; Vinogradov, A.A.; Churakov, A.V.; Ivchenko, P.V. Synthesis of methyl $\beta$-alkylcarboxylates by Pd/diphosphine-catalyzed methoxycarbonylation of methylenealkanes $\mathrm{RCH}_{2} \mathrm{CH}_{2} \mathrm{C}(\mathrm{R})=\mathrm{CH}_{2}$. Appl. Catal. A Gen. 2019, 581, 123-132. [CrossRef]

177. Nifant'ev, I.; Bagrov, V.; Vinogradov, A.; Vinogradov, A.; Ilyin, S.; Sevostyanova, N.; Batashev, S.; Ivchenko, P. Methylenealkane-based Low-Viscosity Ester Oils: Synthesis and Outlook. Lubricants 2020, 8, 50. [CrossRef]

178. Patil, A.O.; Bodige, S. Low Viscosity, Low Volatility Lubricating Oil Basestocks. U.S. Patent 9822326, 21 November 2017.

179. Denis, J. The relationships between structure and rheological properties of hydrocarbons and oxygenated compounds used as base stocks. J. Synth. Lubr. 1984, 1, 201-238. [CrossRef]

180. Lahtela, M.; Pakkanen, T.A.; Nissfolk, F. Molecular Modeling of Poly-a-olefin Synthetic Oils. J. Phys. Chem. 1995, 99, 10267-10271. [CrossRef]

(C) 2020 by the authors. Licensee MDPI, Basel, Switzerland. This article is an open access article distributed under the terms and conditions of the Creative Commons Attribution (CC BY) license (http://creativecommons.org/licenses/by/4.0/). 